\title{
Dual torus fibrations and homological mirror symmetry for $A_{n}$-singularities
}

\author{
KWOKWai Chan and Kazushi Ueda
}

\begin{abstract}
We study homological mirror symmetry for not necessarily compactly supported coherent sheaves on the minimal resolutions of $A_{n}$-singularities. An emphasis is put on the relation with the Strominger-Yau-Zaslow conjecture.
\end{abstract}

\section{Introduction}

Let $Y$ be the affine hypersurface

$$
Y=\left\{(z, u, v) \in \mathbb{C}^{\times} \times \mathbb{C}^{2} \mid u v=z^{-1} f(z)\right\}
$$

where $f(z)=\left(z-a_{0}\right)\left(z-a_{1}\right) \cdots\left(z-a_{n}\right)$ is a polynomial of degree $n+1$ with mutually distinct positive real zeros $0<a_{0}<a_{1}<\cdots<a_{n}$. We equip $Y$ with the symplectic form

$$
\omega=-\left.\frac{\sqrt{-1}}{2}\left(\frac{d z \wedge d \bar{z}}{|z|^{2}}+d u \wedge d \bar{u}+d v \wedge d \bar{v}\right)\right|_{Y}
$$

The projection

$$
\pi: Y \rightarrow \mathbb{C}^{\times}, \quad(z, u, v) \mapsto z
$$

is a conic fibration whose discriminant is given by the zeros $\Delta=\left\{a_{0}, \ldots, a_{n}\right\}$ of the polynomial $f$. Using this, one can show that the map

$$
\rho: Y \rightarrow B, \quad(z, u, v) \mapsto\left(\log |z|, \frac{1}{2}\left(|u|^{2}-|v|^{2}\right)\right)
$$

is a Lagrangian torus fibration over the base $B=\mathbb{R}^{2}$, whose discriminant locus is given by

$$
\Gamma:=\left\{\left(s_{0}, 0\right),\left(s_{1}, 0\right), \ldots,\left(s_{n}, 0\right)\right\},
$$


where $s_{i}=\log a_{i}$ for $i=0, \ldots, n$. Each fiber $L$ of this Lagrangian torus fibration is special in the sense that one has

$$
\left.\mathfrak{I m}\left(\mathrm{e}^{\sqrt{-1} \theta} \Omega\right)\right|_{L}=0
$$

for some $\theta \in \mathbb{R}$, where

$$
\Omega=\operatorname{Res} \frac{d z \wedge d u \wedge d v}{z u v-f(z)}=d \log z \wedge d \log u
$$

is a nowhere-vanishing holomorphic 2-form on $\mathrm{Y}$.

Strominger, Yau and Zaslow (SYZ) [35] conjectured that any CalabiYau manifold admits a special Lagrangian torus fibration, and its mirror is obtained as the dual torus fibration. In this paper, we apply their ideas on the fibration (1.3), which we will refer to as the $S Y Z$ fibration.

Given the SYZ fibration $\rho: Y \rightarrow B$, one can equip the complement $B^{\mathrm{sm}}:=B \backslash \Gamma$ of the discriminant with two tropical affine structures. One is called the symplectic affine structure, and the other is called the complex affine structure. Here a manifold with a tropical affine structure is a manifold which is obtained by gluing open subsets of $\mathbb{R}^{m}$ by the action of the affine linear group $\mathbb{R}^{m} \rtimes G L_{m}(\mathbb{Z})$ (or $\mathbb{R}^{m} \rtimes S L_{m}(\mathbb{Z})$ if the manifold is oriented). An integral affine manifold is the special case when the gluing maps belong to $\mathbb{Z}^{m} \rtimes G L_{m}(\mathbb{Z})$. The symplectic affine structure is defined by first taking a basis $\left\{\gamma_{1}, \gamma_{2}\right\}$ of the space of local sections of the relative homology bundle $\left(R^{1} \rho_{*} \mathbb{Z}\right)^{\vee}$ and integrating the symplectic form $\omega$ along these cycles to obtain 1-forms on $B ; d x_{i}=\int_{\gamma_{i}} \omega$. Local affine coordinate on $B^{\mathrm{sm}}$ are primitives of these 1-forms. The complex affine structure is defined similarly by using $\mathfrak{I} \mathfrak{m}\left(\mathrm{e}^{\sqrt{-1} \theta} \Omega\right)$ instead of $\omega$, and is Legendre dual to the symplectic affine structure [20].

Following earlier works (cf. e.g. [1, 6, 7, 11, 18, 19, 24] and references therein), the mirror $\check{Y}$ of $Y$ is identified in [10] with the complement of an anti-canonical divisor in the minimal resolution of the $A_{n}$-singularity. This mirror $\check{Y}$ admits a special Lagrangian torus fibration, which is an $S Y Z$ mirror in the sense that the symplectic and complex affine structures are interchanged between $Y$ and $\check{Y}$.

Let $E_{1}, \ldots, E_{n} \subset \check{Y}$ be the irreducible components of the exceptional divisor in the minimal resolution. Then there is an isomorphism

$$
\begin{array}{cccc}
\operatorname{deg}: & \operatorname{Pic} \check{Y} & \rightarrow & \mathbb{Z}^{n} \\
\Psi & & \Psi \\
\mathcal{L} & \mapsto & \left(\left.\operatorname{deg} \mathcal{L}\right|_{E_{i}}\right)_{i=1}^{n}
\end{array}
$$


of abelian groups. We write the line bundle associated with $\boldsymbol{d} \in \mathbb{Z}^{n}$ as $\mathcal{L}_{\boldsymbol{d}}$.

Given an SYZ fibration, it is expected that Lagrangian sections of the original manifold and holomorphic line bundles on the mirror manifold are related by a kind of Fourier transform [4,27], which we refer to as the $S Y Z$ transform. We introduce the notion of a strongly admissible path in $\mathbb{C}^{\times} \backslash \Delta$, and associate an exact Lagrangian section $L_{\gamma} \subset Y$ of the SYZ fibration (1.3) to each strongly admissible path. The winding number $\boldsymbol{w}(\gamma)=$ $\left(w_{1}(\gamma), \ldots, w_{n}(\gamma)\right) \in \mathbb{Z}^{n}$ of a strongly admissible path is defined as the intersection numbers with the closed intervals $\left[a_{i-1}, a_{i}\right]$ for $i=1, \ldots, n$.

The first main result in this paper is the following:

Theorem 1.1. For a strongly admissible path $\gamma$, the $S Y Z$ transform of the Lagrangian $L_{\gamma}$ is given by the line bundle $\mathcal{L}_{-\boldsymbol{w}(\gamma)}$.

Next we consider another symplectic manifold defined by

$$
Y^{\prime}=\left\{(z, u, v) \in \mathbb{C}^{\times} \times \mathbb{C}^{2} \mid u v=\frac{1}{z}+z^{n}\right\}
$$

Note that $Y^{\prime}$ is related to $Y$ by moving $a_{i}$ to the roots of unity, and hence is symplectomorphic to it. The map (1.3) gives a special Lagrangian torus fibration on $Y^{\prime}$, whose discriminant consists only of the origin.

The mirror $\check{\mathcal{Y}}$ for $Y^{\prime}$ is the smooth stack obtained by removing an anticanonical divisor from the total space $\mathcal{K}$ of the canonical bundle of the weighted projective line $\mathbb{P}(1, n)$. Although the McKay correspondence [26] gives a derived equivalence

$$
D^{b} \operatorname{coh} \check{Y} \cong D^{b} \operatorname{coh} \check{\mathcal{Y}}
$$

the Picard groups are not isomorphic:

$$
\operatorname{Pic} \check{\mathcal{Y}} \cong \operatorname{Pic} \mathbb{P}(n, 1) \cong \mathbb{Z} ¥ \mathbb{Z}^{n} \cong \operatorname{Pic} \check{Y}
$$

Let $\mathcal{O}_{\check{Y}}(i)$ be the line bundle on $\check{\mathcal{Y}}$ obtained by restricting the pull-back of $\mathcal{O}_{\mathbb{P}(1, n)}(i)$.

Homological mirror symmetry $[22,23]$ for $\mathbb{P}(1, n)$ gives an equivalence

$$
D^{b} \operatorname{coh} \mathbb{P}(1, n) \cong D^{b} \mathfrak{F} \mathfrak{u k} W
$$


where $\mathfrak{F} \mathfrak{u} W$ is a Fukaya category associated with the Lefschetz fibration

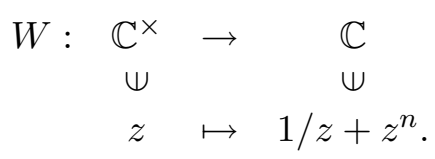

This is a special case of (a generalization to toric stacks of) the work of Abouzaid $[2,3]$ on homological mirror symmetry for toric varieties. There are also works by Fang [15] and Fang, Liu, Treumann and Zaslow $[16,17]$ on homological mirror symmetry for toric varieties, which are also motivated by the SYZ conjecture but different from the work of Abouzaid.

Under the equivalence (1.8), the collection $\left(\mathcal{O}_{\mathbb{P}(1, n)}(i)\right)_{i=0}^{n}$ of line bundles is mapped to Lefschetz thimbles $\left(\Delta_{i}\right)_{i=0}^{n}$. These Lefschetz thimbles can be lifted to Lagrangian sections $\left(L_{i}\right)_{i=0}^{n}$ of the SYZ fibration for $Y^{\prime}$. Let $\mathcal{W}^{\prime}$ be the full subcategory of the wrapped Fukaya category of $Y^{\prime}$ consisting of $\left(L_{i}\right)_{i=0}^{n}$.

The second main result in this paper is the following:

Theorem 1.2. There is an equivalence

$$
D^{b} \mathcal{W}^{\prime} \cong D^{b} \operatorname{coh} \check{\mathcal{Y}}
$$

of triangulated categories sending $L_{i}$ to $\mathcal{O}_{\check{\mathcal{Y}}}(i)$ for $i=0, \ldots, n$.

The proof is based on an analysis of the behavior of the wrapped Fukaya category under suspension, and depends heavily on the work of Pascaleff [29]. We expect that $\left(L_{i}\right)_{i=0}^{n}$ generates the wrapped Fukaya category, so that the left-hand side of (1.9) is the whole wrapped Fukaya category.

Theorems 1.1 and 1.2 are compatible in the following sense: There exists a symplectomorphism $Y \stackrel{\sim}{\rightarrow} Y^{\prime}$ which induces an equivalence

$$
D^{b} \mathcal{W} \stackrel{\sim}{\rightarrow} D^{b} \mathcal{W}^{\prime}
$$

so that the Lagrangians $\left(L_{i}\right)_{i=0}^{n}$ in $Y^{\prime}$ are images of Lagrangians $\left(L_{\gamma_{i}}\right)_{i=0}^{n}$ in $Y$ associated with certain strongly admissible paths $\gamma_{0}, \gamma_{1}, \ldots, \gamma_{n}$ in $\mathbb{C}^{\times} \backslash \Delta$. One can then choose a derived equivalence

$$
D^{b} \operatorname{coh} \check{\mathcal{Y}} \stackrel{\sim}{\longrightarrow} D^{b} \operatorname{coh} \check{Y}
$$

in such a way that the images of $\left(L_{\gamma_{i}}\right)_{i=0}^{n}$ under the composition

$$
D^{b} \mathcal{W} \stackrel{\sim}{\longrightarrow} D^{b} \mathcal{W}^{\prime} \stackrel{\sim}{\longrightarrow} D^{b} \operatorname{coh} \check{\mathcal{Y}} \stackrel{\sim}{\longrightarrow} D^{b} \operatorname{coh} \check{Y}
$$


of equivalences are precisely given by their SYZ transforms described in Theorem 1.1. This shows that homological mirror symmetry for non-compact branes is realized by SYZ just as for compact branes [10].

This paper is organized as follows: In Section 2, we briefly recall the SYZ construction of the mirror manifold $\check{Y}$ from [10, Section 3]. In Section 3, we introduce the notion of a strongly admissible path to which we associate a noncompact Lagrangian submanifold in $(Y, \omega)$, which is a section of the SYZ fibration $\rho$. In Section 4, we describe holomorphic line bundles on $\check{Y}$ obtained as SYZ transforms of noncompact Lagrangian submanifolds associated with strongly admissible paths and prove Theorem 1.1. The proof of Theorem 1.2 is given in Section 5 .

\section{SYZ mirror symmetry}

We start with the Hamiltonian $S^{1}$-action on $(Y, \omega)$ :

$$
\mathrm{e}^{2 \pi \sqrt{-1} t} \cdot(u, v, z)=\left(\mathrm{e}^{2 \pi \sqrt{-1} t} u, \mathrm{e}^{-2 \pi \sqrt{-1} t} v, z\right)
$$

whose moment map is given by

$$
\mu(u, v, z)=\frac{1}{2}\left(|u|^{2}-|v|^{2}\right) .
$$

Let $B:=\mathbb{R}^{2}$. Then the map $\rho: Y \rightarrow B$ defined by

$$
\rho(u, v, z)=(\log |z|, \mu)=\left(\log |z|, \frac{1}{2}\left(|u|^{2}-|v|^{2}\right)\right)
$$

is a Lagrangian torus fibration on $Y$, whose discriminant locus is given by the finite set

$$
\Gamma:=\left\{\left(s_{0}, 0\right),\left(s_{1}, 0\right), \ldots,\left(s_{n}, 0\right)\right\} \subset B,
$$

where we denote $s_{i}:=\log a_{i}$ for $i=0, \ldots, n$. This is usually called an $S Y Z$ fibration of $(Y, \omega)$.

Let $B^{\mathrm{sm}}:=B \backslash \Gamma$ be the smooth locus. Then the fiber $T_{s, \lambda}$ over a point $(s, \lambda) \in B^{\mathrm{sm}}$ is a smooth Lagrangian torus in $Y$, and each of the fiber $T_{s_{i}, 0}$ over $\left(s_{i}, 0\right) \in \Gamma$ is singular with one nodal singularity. Furthermore, the locus of Lagrangian torus fibers which bound nonconstant holomorphic disks is given by a union of vertical lines:

$$
H:=\left\{(s, \lambda) \in B \mid s=s_{i} \text { for some } i=0,1, \ldots, n\right\}
$$

Each connected component of $H$ is called a wall in $B$. 
The Lagrangian torus fibration $\rho: Y \rightarrow B$ induces an integral affine structure on $B^{\mathrm{sm}}$, called the symplectic affine structure. As we have mentioned, integrality of the affine structure means that the transition maps between charts on $B^{\mathrm{sm}}$ are elements of the integral affine linear group

$$
\operatorname{Aff}\left(\mathbb{Z}^{2}\right):=\mathrm{GL}_{2}(\mathbb{Z}) \rtimes \mathbb{Z}^{2} .
$$

This ensures that $\Lambda \subset T B^{\mathrm{sm}}$, the family of lattices locally generated by $\partial / \partial x_{1}, \partial / \partial x_{2}$ where $x_{1}, x_{2}$ are local affine coordinates on $B^{\mathrm{sm}}$, is well-defined.

The SYZ conjecture [35] suggested that a mirror of $(Y, \omega)$ can be constructed by fiberwise-dualizing an SYZ fibration on $(Y, \omega)$. More precisely, one defines the semi-flat mirror of $(Y, \omega)$ as the moduli space $\check{Y}_{0}$ of pairs $\left(T_{s, \lambda}, \nabla\right)$ where $\nabla$ is a flat $U(1)$-connection (up to gauge equivalence) on the trivial line bundle $\mathbb{C}:=\mathbb{C} \times T_{s, \lambda}$ over a smooth Lagrangian torus fiber $T_{s, \lambda}$. Topologically, $\check{Y}_{0}$ is simply the quotient $T B^{\mathrm{sm}} / \Lambda$ of the tangent bundle $T B^{\text {sm }}$ by $\Lambda$. This is naturally a complex manifold where the local complex coordinates are given by exponentiations of complexifications of local affine coordinates on $B^{\mathrm{sm}}$ (while the quotient $T^{*} B^{\mathrm{sm}} / \Lambda^{\vee}$ of the cotangent bundle $T^{*} B^{\mathrm{sm}}$ by the dual lattice $\Lambda^{\vee}$, which is canonically a symplectic manifold, is contained in $Y$ as an open dense subset such that the restriction of $\omega$ to it gives the canonical symplectic structure). However, this is not quite the correct mirror manifold because the natural complex structure on $\check{Y}_{0}$ is not globally defined due to nontrivial monodromy of the affine structure around each singular point $\left(s_{i}, 0\right) \in \Gamma$.

A more concrete description of this phenomenon is as follows. For $i=0,1, \ldots, n$, consider the strip $B_{i}:=\left(s_{i-1}, s_{i+1}\right) \times \mathbb{R}$ (where we set $s_{-1}:=$ $-\infty$ and $\left.s_{n+1}:=+\infty\right)$. A covering of $B^{\mathrm{sm}}$ is given by the open sets

$$
U_{i}:=B_{i} \backslash\left[s_{i}, s_{i+1}\right) \times\{0\}, \quad V_{i}:=B_{i} \backslash\left(s_{i-1}, s_{i}\right] \times\{0\} .
$$

$i=0,1, \ldots, n$. The intersection $U_{i} \cap V_{i}$ consists of two connected components:

$$
U_{i} \cap V_{i}=B_{i}^{+} \cup B_{i}^{-},
$$

where $B_{i}^{+}$(resp. $B_{i}^{-}$) corresponds to the component in which $\lambda>0$ (resp. $\lambda<0)$.

On $U_{i}$ (resp. $V_{i}$ ), denote by $u_{i}$ (resp. $v_{i+1}$ ) the exponentiationof the complexification of the left-pointing (resp. right-pointing) affine coordinate. (Our convention is that for a real number $x \in \mathbb{R}$, its complexification is given by $-x+\sqrt{-1} y$.) Also, denote by $w$ the exponentiation of the complexification of the upward-pointing affine coordinate. See figure 1. 


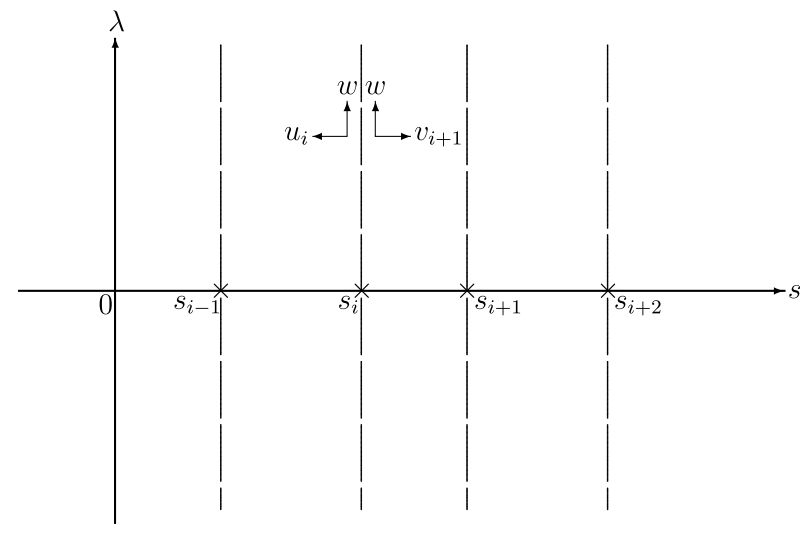

Figure 1: The base affine manifold $B$.

Now $w$ is a globally defined coordinate. Geometrically, it can be written as

$$
w\left(T_{s, \lambda}, \nabla\right)= \begin{cases}\exp \left(-\int_{\alpha} \omega\right) \operatorname{hol}_{\nabla}(\partial \alpha) & \text { for } \lambda \geq 0, \\ \exp \left(\int_{\alpha} \omega\right) \operatorname{hol}_{\nabla}(-\partial \alpha) & \text { for } \lambda \leq 0,\end{cases}
$$

where $\alpha \in \pi_{2}\left(Y, T_{s_{i}, \lambda}\right)$ is the class of the holomorphic disk in $Y$ bounded by $T_{s_{i}, \lambda}$ for some $i=0,1, \ldots, n$, and $\operatorname{hol}_{\nabla}(\partial \alpha)$ is the holonomy of the flat $U(1)$ connection $\nabla$ around the boundary $\partial \alpha \in \pi_{1}\left(T_{s_{i}, \lambda}\right)$. The class $\alpha$ changes to $-\alpha$ when one moves from $\lambda>0$ to $\lambda<0$.

But the other coordinates $u_{i}$ and $v_{i+1}$ are not globally defined: since the monodromy of the affine structure going counter-clockwise around $\left(s_{i}, 0\right) \in \Gamma$ is given by the matrix

$$
\left(\begin{array}{ll}
1 & 1 \\
0 & 1
\end{array}\right)
$$

the coordinates $\left(u_{i}, w\right)$ and $\left(v_{i+1}, w\right)$ glue on $U_{i} \cap V_{i}$ according to

$$
\begin{gathered}
u_{i}=v_{i+1}^{-1} \text { on } B_{i}^{+}, \\
u_{i}=v_{i+1}^{-1} w \text { on } B_{i}^{-} .
\end{gathered}
$$

Hence, the monodromy of the complex coordinates going counter-clockwise around the point $\left(s_{i}, 0\right)$ is nontrivial and given by

$$
u_{i} \mapsto u_{i} w, \quad v_{i+1}^{-1} \mapsto v_{i+1}^{-1} w .
$$

In particular, these complex coordinates on $T B_{i} / \Lambda$ do not form a globally defined complex structure on $\check{Y}_{0}$. 
The examples in $[1,6,7,11]$ suggest the following construction (we should mention that these are all special cases of the constructions in Kontsevich and Soibelman [25] and Gross and Siebert [19], but those general constructions largely ignore the symplectic aspects and hence Lagrangian torus fibration structures - they build the mirror family directly from tropical data in the base $B$ ): in order to get the genuine mirror manifold, we need to modify the gluing of complex charts on $\check{Y}_{0}$ by quantum corrections from disk instantons

$$
\begin{aligned}
& u_{i}=v_{i+1}^{-1}+v_{i+1}^{-1} w=v_{i+1}^{-1}(1+w) \quad \text { on } B_{i}^{+}, \\
& u_{i}=v_{i+1}^{-1} w+v_{i+1}^{-1}=v_{i+1}^{-1} w\left(1+w^{-1}\right) \quad \text { on } B_{i}^{-} .
\end{aligned}
$$

Geometrically, the term $v_{i+1}^{-1} w$ in the first formula (2.1) should be viewed as multiplying $v_{i+1}^{-1}$ by $w$ where $w$ corresponds to the unique nonconstant holomorphic disk bounded by the Lagrangian torus $T_{0, \lambda}$ whose area is given by

$$
\lambda=-\log |w|>0 \text {. }
$$

This means that we are correcting the term $v_{i+1}^{-1}$ by adding the contribution from the holomorphic disk bounded by $T_{0, \lambda}$ when we cross the upper half of the wall $\left\{s_{i}\right\} \times \mathbb{R} \subset H$. In the same way, the term $v_{i+1}^{-1}$ in the second formula $(2.2)$ is given by multiplying $v_{i+1}^{-1} w$ by $w^{-1}$ where $w^{-1}$ now corresponds to the unique nonconstant holomorphic disk bounded by the Lagrangian torus $T_{0, \lambda}$ whose area is equal to

$$
-\lambda=\log |w|=-\log \left|w^{-1}\right|>0 .
$$

So we are correcting $v_{i+1}^{-1} w$ by the disk bounded by $T_{0, \lambda}$ when we cross the lower half of the wall $\left\{s_{i}\right\} \times \mathbb{R} \subset H$.

The formulas $(2.1),(2.2)$ can actually be interpreted as wall-crossing formulas for the counting of Maslov index two holomorphic disks in $Y$ bounded by Lagrangian torus fibers. To see this, we need to partially compactify $Y$ by allowing $z$ to take values in $\mathbb{C}$ and replacing $\rho$ by the map

$$
(u, v, z) \mapsto(|z|, \mu)=\left(|z|, \frac{1}{2}\left(|u|^{2}-|v|^{2}\right)\right) .
$$

Then the base becomes an affine manifold with both singularities and boundary: it is a right half-space $\bar{B}$ in $\mathbb{R}^{2}$, and the boundary $\partial \bar{B}$ corresponds to the divisor in $Y$ defined by $z=0$. In this situation, each of the local coordinates 
$u_{i}, v_{i+1}^{-1}$ and $v_{i+1}^{-1} w$ can be expressed as in the form

$$
\exp \left(-\int_{\beta} \omega\right) \operatorname{hol}_{\nabla}(\partial \beta),
$$

for a suitable relative homotopy class $\beta \in \pi_{2}\left(Y, T_{s, \lambda}\right)$ of Maslov index two (cf. Auroux $[6,7]$ ).

In any case, the modification of gluing cancels the monodromy and defines global complex coordinates $u_{i}, v_{i}, w$ on $T B_{i} / \Lambda$ (topologically, $T B_{i} / \Lambda$ $\cong\left(\mathbb{C}^{\times}\right)^{2}$ ) related by

$$
u_{i}=v_{i}^{-1}, \quad u_{i+1}=v_{i+1}^{-1} \text { and } u_{i} v_{i+1}=1+w
$$

The instanton-corrected mirror $\check{Y}$ is then obtained by gluing together the pieces $T B_{i} / \Lambda(i=0,1, \ldots, n)$ according to (2.3). On the intersection $\left(T B_{i-1} / \Lambda\right) \cap\left(T B_{i} / \Lambda\right)$, we have

$$
u_{i}=v_{i}^{-1}, \quad u_{i} v_{i+1}=1+w=u_{i-1} v_{i} .
$$

We remark that, by our definition, the mirror manifold $\check{Y}$ will have "gaps" because for instance $u, v, w$ are $\mathbb{C}^{\times}$-valued. A natural way to "complete" the mirror manifold and fill out its gaps is by rescaling the symplectic structure of $Y$ and performing analytic continuation; see [6, Section 4.2] for a discussion of this "renormalization" process.

Finally, one observes that this is precisely the gluing of complex charts in the toric resolution $X \rightarrow \mathbb{C}^{2} / \mathbb{Z}_{n+1}$ of the $A_{n}$-singularity. We thus conclude that the mirror is precisely given by the complex manifold

$$
\check{Y}:=X \backslash D
$$

where $X=X_{\Sigma}$ is the toric surface defined by the 2-dimensional fan $\Sigma \in \mathbb{R}^{2}$ generated by

$$
\left\{v_{i}:=(i-1,1) \in N \mid i=0,1, \ldots, n+1\right\},
$$

and $D$ is the hypersurface in $X_{\Sigma}$ defined by $h:=\chi^{(0,1)}=1$. Here $h: X \rightarrow \mathbb{C}$ is the holomorphic function whose zero locus is the union of all the toric divisors in $X$. For $i=1, \ldots, n$, if we let $S_{i}$ denote the interval $\left(s_{i-1}, s_{i}\right) \times$ $\{0\} \subset B$, then (the closure of) the quotient $T S_{i} / T S_{i} \cap \Lambda$ in $\check{Y}$ is one of the $n$ exceptional divisors in $X_{\Sigma}$, each of which is a $(-2)$-curve. We denote this exceptional divisor by $E_{i} \subset \check{Y}$. 
Let $M=\operatorname{Hom}(N, \mathbb{Z})$ be the lattice of characters of the dense torus of $X_{\Sigma}$ and $\Sigma(1)=\left\{v_{0}, \ldots, v_{n+1}\right\}$ be the set of one-dimensional cones of the fan $\Sigma$. If we write the toric divisor on $X_{\Sigma}$ corresponding to $v_{i} \in \Sigma(1)$ as $D_{i}$, then we have $D_{i}=E_{i}$ for $i=1, \ldots, n$, and $D_{0}$ and $D_{n+1}$ are non-compact divisors. One can easily show from the divisor sequence

$$
0 \rightarrow M \rightarrow \mathbb{Z}^{\Sigma(1)} \rightarrow \operatorname{Pic} X_{\Sigma} \rightarrow 0
$$

that $\operatorname{Pic} X_{\Sigma}$ is generated by $\mathcal{O}\left(D_{i}\right)$ for $i=0, \ldots, n-1$, and the map

$$
\begin{aligned}
& \operatorname{deg}: \operatorname{Pic} X_{\Sigma} \quad \rightarrow \quad \mathbb{Z}^{n} \\
& \Psi \quad \psi \\
& \mathcal{L} \quad \mapsto \quad\left(\left.\operatorname{deg} \mathcal{L}\right|_{D_{i}}\right)_{i=1}^{n}
\end{aligned}
$$

is an isomorphism of abelian groups. Since $\check{Y}=X_{\Sigma} \backslash D$ is the complement of a principal divisor, the restriction map Pic $X_{\Sigma} \rightarrow \operatorname{Pic} \check{Y}$ and hence the map (1.5) is an isomorphism of abelian groups.

\section{Lagrangian submanifolds fibered over paths}

Consider the projection map

$$
\pi: Y \rightarrow \mathbb{C}^{\times}, \quad(u, v, z) \mapsto z .
$$

Each fiber is a conic $\left\{(u, v) \in \mathbb{C}^{2} \mid u v=z^{-1} f(z)\right\}$ which degenerates to the cone $u v=0$ over a zero of the polynomial $f$. Recall that we denote by

$$
\Delta=\left\{a_{0}, a_{1}, \ldots, a_{n}\right\}
$$

the set of zeros of the polynomial $f(z)$, which we have assumed to be real and positive, and in the ordering

$$
0<a_{0}<a_{1}<\ldots<a_{n}
$$

The symplectic fibration $\pi: Y \rightarrow \mathbb{C}^{\times}$induces a connection on the tangent bundle of $Y$ where the horizontal subspace at $y \in Y_{0}$ is given by the symplectic orthogonal complement to the vertical subspace:

$$
H_{y}:=\operatorname{Ker}\left(d \pi_{y}\right)^{\perp_{\omega}} .
$$

Given a smooth embedded path $\gamma: I \rightarrow \mathbb{C}^{\times} \backslash \Delta$ where $I \subset \mathbb{R}$ is an interval, parallel transport with respect to the above connection along $\gamma$ yields 
symplectomorphisms between smooth fibers

$$
\tau_{t_{1}}^{t_{2}}: \pi^{-1}\left(\gamma\left(t_{1}\right)\right) \rightarrow \pi^{-1}\left(\gamma\left(t_{2}\right)\right)
$$

for $t_{1}<t_{2} \in I$. These symplectomorphisms are $S^{1}$-equivariant since the $S^{1}$ action is Hamiltonian. The vanishing cycle $V_{t}$ in a smooth fiber $\pi^{-1}(\gamma(t))$ is given by its equator $\mu=0$ (i.e. $|u|=|v|$ ). Vanishing cycles are invariant under parallel transport, so that the Lefschetz thimble $L_{\gamma}$ along $\gamma$, given by the union of all vanishing cycles $V_{t} \subset \pi^{-1}(\gamma(t))$ for $t \in I$, is a Lagrangian submanifold in $(Y, \omega)$. If $\gamma$ is a matching path, i.e., a path connecting two critical values of $\pi$ in $\mathbb{C}^{\times}$, then $L_{\gamma}$ is a Lagrangian sphere in $(Y, \omega)$.

Other Lagrangian submanifolds can be constructed in a similar way, as in the work of Pascaleff [29].

Definition 3.1. Let $\gamma: \mathbb{R} \rightarrow \mathbb{C}^{\times} \backslash \Delta$ be a smooth embedded path. We call the path $\gamma$ admissible if the following conditions are satisfied:

(1) (boundary conditions) $\lim _{t \rightarrow-\infty}|\gamma(t)|=0$ and $\lim _{t \rightarrow \infty}|\gamma(t)|=\infty$.

(2) $\gamma$ intersects transversally with each of the line segments $\epsilon_{i}:=\left[a_{i-1}, a_{i}\right]$ for $i=1, \ldots, n$.

Given an admissible path $\gamma: \mathbb{R} \rightarrow \mathbb{C}^{\times}$, we fix $t_{0} \in \mathbb{R}$ and choose a Lagrangian cycle $C_{0}$ in the conic fiber $\pi^{-1}\left(\gamma\left(t_{0}\right)\right)$. Then the submanifold $L_{\gamma, C_{0}}$ contained inside $\pi^{-1}(\gamma)$ swept out by the parallel transport of $C_{0}$ along $\gamma$ is a Lagrangian submanifold in $(Y, \omega)$ (cf. [6, Section 5.1] and [29, Section $3.3])$.

Definition 3.2. Let $\gamma: \mathbb{R} \rightarrow \mathbb{C}^{\times} \backslash \Delta$ be an admissible path. For $i=1, \ldots$, $n$, we define the ith winding number $w_{i}(\gamma)$ of $\gamma$ to be the topological intersection number between $\gamma(\mathbb{R})$ and the line segment $\epsilon_{i}=\left[a_{i-1}, a_{i}\right] \subset \mathbb{C}^{\times}$.

Notice that the winding numbers of $\gamma$ are invariant when we deform $\gamma$ in a fixed homotopy class relative to the boundary conditions $\lim _{t \rightarrow-\infty}|\gamma(t)|=0$ and $\lim _{t \rightarrow \infty}|\gamma(t)|=\infty$. In particular, we can deform $\gamma$ so that $\gamma(t)$ lies on the negative real axis for $t<-T$ for some fixed positive $T$. Then we consider the Lagrangian (real locus)

$$
C_{t}:=\left\{(u, v, z) \in \pi^{-1}(\gamma(t)) \mid u, v \in \mathbb{R}\right\} .
$$

in the conic fiber $\pi^{-1}(\gamma(t))$ for each $t<-T$, which is 'dual' to the vanishing cycle $V_{t}$. Note that $C_{t}$ is invariant under symplectic parallel transport for 
all $t<-T$, meaning that

$$
\tau_{t_{1}}^{t_{2}}\left(C_{t_{1}}\right)=C_{t_{2}}
$$

for all $t_{1}<t_{2}<-T$. Let $L_{\gamma}$ be the submanifold in $Y$ swept out by parallel transport of $C_{t}(t<-T)$ :

$$
L_{\gamma}:=\bigcup_{t \in(-\infty,-T)} C_{t} \cup \bigcup_{t \in[-T, \infty)} \tau_{-T}^{t}\left(C_{-T}\right) .
$$

This defines a Lagrangian submanifold in $(Y, \omega)$ which is homeomorphic to $\mathbb{R}^{2}$. Note that the curve $C_{-T}:=\left\{(u, v, z) \in \pi^{-1}(\gamma(-T)) \mid u, v \in \mathbb{R}\right\}$ in the conic fiber $\pi^{-1}(\gamma(-T))$ is being twisted by Dehn twists in vanishing cycles (i.e. the equator $\lambda=0$ in a fiber of $\pi: Y \rightarrow \mathbb{C}^{\times}$) as one goes along $\gamma$.

Furthermore, given an admissible path $\gamma$, we can deform it (again with respect to the boundary conditions $\lim _{t \rightarrow-\infty}|\gamma(t)|=0$ and $\lim _{t \rightarrow \infty}|\gamma(t)|=$ $\infty)$ so that the following condition is satisfied:

Definition 3.3. An admissible path $\gamma: \mathbb{R} \rightarrow \mathbb{C}^{\times} \backslash \Delta$ is strongly admissible if the modulus $|\gamma|: \mathbb{R} \rightarrow \mathbb{R}^{>0}$ is strictly increasing.

Proposition 3.4. Let $\gamma: \mathbb{R} \rightarrow \mathbb{C}^{\times}$be a strongly admissible path. Then the Lagrangian submanifold $L_{\gamma}$ is a section of the $S Y Z$ fibration $\rho: Y \rightarrow B$.

Proof. By definition, $L_{\gamma}$ is invariant under parallel transport, so it is a Lagrangian submanifold in $(Y, \omega)$. Moreover, it is clear that the moment map $\mu$ when restricted to $C_{t}$ for $t<-T$ is a one-to-one map. Since the symplectomorphisms $\tau_{-T}^{t}$ are all $S^{1}$-equivariant, the value of $\mu$ is preserved under parallel transport. Thus the restriction of $\mu$ to $\tau_{-T}^{t}\left(C_{-T}\right)$ remains a one-to-one map. Together with the condition that $|\gamma(t)|$ is strictly increasing, this implies that $L_{\gamma}$ intersects each fiber $T_{s, \lambda}$ of the SYZ fibration $\rho: Y \rightarrow B$ at one point. Hence $L_{\gamma}$ is a section of $\rho: Y \rightarrow B$.

By deforming $\gamma$ in a fixed homotopy class if necessary, one can furthermore assume that a strongly admissible path $\gamma: \mathbb{R} \rightarrow \mathbb{C}^{\times} \backslash \Delta$ satisfies $|\gamma(s)|=\mathrm{e}^{s}$ for any $s \in \mathbb{R}$ and $\gamma\left(s_{i}\right)=-a_{i}$ for $i=0, \ldots, n$ (recall that $s_{i}=$ $\log a_{i}$ for each $i$ ). Then there exists a unique lift $\widetilde{\gamma}: \mathbb{R} \rightarrow \mathbb{R}$ of $\gamma /|\gamma|: \mathbb{R} \rightarrow S^{1}$ such that $\widetilde{\gamma}\left(s_{0}\right)=0$, and the winding numbers of $\gamma$ can be computed as

$$
w_{i}(\gamma)=\frac{1}{2 \pi}\left(\tilde{\gamma}\left(s_{i}\right)-\tilde{\gamma}\left(s_{i-1}\right)\right)
$$

for $i=1, \ldots, n$. 
As an example, consider the path

$$
\gamma_{0}: \mathbb{R} \rightarrow \mathbb{C}^{\times}, \quad t \mapsto-e^{t}
$$

which is clearly strongly admissible. The corresponding Lagrangian submanifold $L_{0}:=L_{\gamma_{0}}$ is simply the Cartesian product of the real locus $C_{s_{0}}$ inside the conic fiber $C_{s_{0}}=\pi^{-1}\left(-a_{0}\right)$ with the negative real axis (which is the image $\gamma_{0}(\mathbb{R})$ of the path). As a section of the SYZ fibration $\rho: Y \rightarrow B$, the Lagrangian $L_{0}$ can be explicitly written as

$$
\sigma: B \rightarrow Y, \quad(s, \lambda) \mapsto(u(s, \lambda), v(s, \lambda), z(s, \lambda))
$$

where

$$
\begin{aligned}
& u(s, \lambda)=\sqrt{\sqrt{\lambda^{2}+\left|f\left(-\mathrm{e}^{s}\right)\right|^{2}}+\lambda} \\
& v(s, \lambda)=(-1)^{n-1} \sqrt{\sqrt{\lambda^{2}+\left|f\left(-\mathrm{e}^{s}\right)\right|^{2}}-\lambda} \\
& z(s, \lambda)=-\mathrm{e}^{s} .
\end{aligned}
$$

\section{SYZ transforms}

Consider $\Lambda^{\vee} \subset T^{*} B^{\mathrm{sm}}$, the family of lattices locally generated by $d x_{1}, d x_{2}$. As before, $x_{1}, x_{2}$ here denote local affine coordinates on $B^{\mathrm{sm}}$. As in [10], we will assume that $x_{2}=-\lambda$ is the globally defined coordinate. Let

$$
\omega_{0}:=d x_{1} \wedge d \xi_{1}+d x_{2} \wedge d \xi_{2}
$$

be the canonical symplectic structure on the quotient $T^{*} B^{\mathrm{sm}} / \Lambda^{\vee}$ of the cotangent bundle $T^{*} B^{\mathrm{sm}}$ by $\Lambda^{\vee}$, where $\left(\xi_{1}, \xi_{2}\right)$ are fiber coordinates on $T^{*} B^{\mathrm{sm}}$ so that $\left(x_{1}, x_{2}, \xi_{1}, \xi_{2}\right) \in T^{*} B^{\mathrm{sm}}$ denotes the cotangent vector $\xi_{1} d x_{1}+$ $\xi_{2} d x_{2}$ at the point $\left(x_{1}, x_{2}\right) \in B^{\mathrm{sm}}$. In the previous section, we have constructed a global Lagrangian section $L_{0}$ of the SYZ fibration $\rho: Y \rightarrow B$. Then a theorem of Duistermaat [13] says that there exists a fiber-preserving symplectomorphism

$$
\Theta:\left(T^{*} B^{\mathrm{sm}} / \Lambda^{\vee}, \omega_{0}\right) \stackrel{\cong}{\longrightarrow}\left(\rho^{-1}\left(B^{\mathrm{sm}}\right), \omega\right),
$$

so that $L_{0}$ corresponds to the zero section of $T^{*} B^{\mathrm{sm}} / \Lambda^{\vee}$.

This symplectomorphism can be constructed as follows. Let $b \in B^{\mathrm{sm}}$. Then for every $\alpha \in T_{b}^{*} B^{\mathrm{sm}}$, we can associate a vector field $v_{\alpha}$ on the fiber 
$\rho^{-1}(b)$ by

$$
\iota_{v_{\alpha}} \omega=\rho^{*} \alpha .
$$

Let $\phi_{\alpha}^{\tau}$ be the flow of $v_{\alpha}$ at time $\tau \in \mathbb{R}$. Then we define an action $\theta_{\alpha}$ of $\alpha$ on $\rho^{-1}(b)$ by the time-1 flow

$$
\theta_{\alpha}(y)=\phi_{\alpha}^{1}(y), \quad y \in \rho^{-1}(b)
$$

Now the covering map

$$
T^{*} B^{\mathrm{sm}} \rightarrow \rho^{-1}\left(B^{\mathrm{sm}}\right), \quad \alpha \mapsto \theta_{\alpha}(\sigma(\operatorname{pr}(\alpha)))
$$

where pr: $T^{*} B^{\mathrm{sm}} \rightarrow B^{\mathrm{sm}}$ denotes the projection map, induces a symplectomorphism $\Theta: T^{*} B^{\mathrm{sm}} / \Lambda^{\vee} \rightarrow \rho^{-1}\left(B^{\mathrm{sm}}\right)$.

Now let $y_{1}, y_{2}$ be the fiber coordinates on $T B^{\mathrm{sm}}$ dual to $\xi_{1}, \xi_{2}$, i.e. $\left(x_{1}, x_{2}, y_{1}, y_{2}\right) \in T B^{\mathrm{sm}}$ denotes the tangent vector $y_{1} \partial / \partial x_{1}+y_{2} \partial / \partial x_{2}$ at the point $\left(x_{1}, x_{2}\right) \in B^{\mathrm{sm}}$. Given a strongly admissible path $\gamma: \mathbb{R} \rightarrow \mathbb{C}^{\times}$, the noncompact Lagrangian submanifold $L_{\gamma}$ is a section of the SYZ fibration $\rho: Y \rightarrow B$ by Proposition 3.4 (and as we mentioned before, every admissible path can be deformed to a strongly admissible one). Via the symplectomorphism $\Theta$, we can write $L_{\gamma}$ in the form

$$
\begin{aligned}
L_{\gamma} & =\left\{\left(x_{1}, x_{2}, \xi_{1}, \xi_{2}\right) \in T^{*} B^{\mathrm{sm}} / \Lambda^{\vee} \mid\left(x_{1}, x_{2}\right) \in B^{\mathrm{sm}},\right. \\
\xi_{j} & \left.=\xi_{j}\left(x_{1}, x_{2}\right) \text { for } j=1,2\right\}
\end{aligned}
$$

where $\xi_{j}=\xi_{j}\left(x_{1}, x_{2}\right), j=1,2$, are smooth functions on $B^{\mathrm{sm}}$. Since $L_{\gamma}$ is Lagrangian, the functions $\xi_{1}, \xi_{2}$ satisfy

$$
\frac{\partial \xi_{j}}{\partial x_{l}}=\frac{\partial \xi_{l}}{\partial x_{j}}
$$

for $j, l=1,2($ see $[4,10,27])$.

Lying at the basis of the SYZ proposal [35] is the fact that the dual $T^{*}$ of a torus $T$ can be viewed as the moduli space of flat $U(1)$-connections on the trivial line bundle $\mathbb{C}:=\mathbb{C} \times T$ over $T$. So a Lagrangian section $L=\left\{(x, \xi(x)) \in T^{*} U / T^{*} U \cap \Lambda^{\vee} \mid x \in U\right\}$ over an open set $U \subset B^{\text {sm }}$ corresponds to a family of connections $\left\{\nabla_{\xi(x)} \mid x \in U\right\}$ patching together to give a $U(1)$-connection which can locally be written as

$$
\check{\nabla}_{U}=d+2 \pi \sqrt{-1}\left(\xi_{1} d y_{1}+\xi_{2} d y_{2}\right)
$$


over $T U / T U \cap \Lambda \subset \check{Y}$. As shown in $[4,27]$ (see also [10, Section 2]), the $(0,2)$-part $F^{(0,2)}$ (and also $(2,0)$-part $\left.F^{(2,0)}\right)$ of the curvature two form of $\check{\nabla}_{U}$ is trivial.

Recall that a covering of $B^{\mathrm{sm}}$ is given by the open sets

$$
U_{i}:=B_{i} \backslash\left[s_{i}, s_{i+1}\right) \times\{0\}, \quad V_{i}:=B_{i} \backslash\left(s_{i-1}, s_{i}\right] \times\{0\} .
$$

for $i=0,1, \ldots, n$. Now, the restriction of the Lagrangian section $L_{\gamma}$ to each $U_{i}$ (resp. $\left.V_{i}\right)$ is transformed to a $U(1)$-connection $\check{\nabla}_{U_{i}}$ over $T U_{i} / T U_{i} \cap \Lambda$ (resp. $\check{\nabla}_{V_{i}}$ over $T V_{i} / T V_{i} \cap \Lambda$ ). These connections can be glued together according to the gluing formulas $(2.1),(2.2)$. Since the $(0,2)$-part $F^{(0,2)}$ of the curvature two form vanishes, this defines a holomorphic line bundle $\mathcal{L}_{\gamma}$ over $\check{Y}$.

Definition 4.1. Let $\gamma: \mathbb{R} \rightarrow \mathbb{C}^{\times}$be a strongly admissible path and $L_{\gamma}$ be the noncompact Lagrangian submanifold in $(Y, \omega)$ associated to $\gamma$. We define the SYZ transform of $L_{\gamma}$ to be the holomorphic line bundle $\mathcal{L}_{\gamma}$ over $\check{Y}$, i.e.

$$
\mathcal{F}\left(L_{\gamma}\right):=\mathcal{L}_{\gamma}
$$

Notice that the isomorphism class of $\mathcal{L}_{\gamma}$ is unchanged when we deform $L_{\gamma}$ in a fixed Hamiltonian isotopy class (or deforming $\gamma$ is a fixed homotopy class relative to the boundary conditions $\lim _{t \rightarrow-\infty}|\gamma(t)|=0$ and $\lim _{t \rightarrow \infty}|\gamma(t)|=$ $\infty)$. Henceforth, we will regard this as defining the SYZ transform of the Hamiltonian isotopy class of the Lagrangian submanifold $L_{\gamma} \subset Y$ as an isomorphism class of holomorphic line bundle over $\check{Y}$.

As an immediate example, the SYZ transform of the zero section $L_{0}=$ $L_{\gamma_{0}}$ gives the structure sheaf $\mathcal{O}_{\check{Y}}$ over $\check{Y}$.

The main goal of this section is to compute (the isomorphism class of) the line bundle $\mathcal{L}_{\gamma}$ in terms of the winding numbers of the path $\gamma$. To begin with, note that the isomorphism class of a line bundle over $\check{Y}$ is determined by the degrees of its restrictions to the exceptional divisors $E_{i}$ for $i=1, \ldots, n$. Given integers $d_{1}, \ldots, d_{n} \in \mathbb{Z}$, let us denote by $\mathcal{L}_{d_{1}, \ldots, d_{n}}$ the line bundle on $\check{Y}$ such that $\left.\operatorname{deg} \mathcal{L}_{d_{1}, \ldots, d_{n}}\right|_{E_{i}}=d_{i}$.

Now, given a Lagrangian section

$$
\begin{aligned}
L_{\gamma} & =\left\{\left(x_{1}, x_{2}, \xi_{1}, \xi_{2}\right) \in T^{*} B^{\mathrm{sm}} / \Lambda^{\vee} \mid\left(x_{1}, x_{2}\right) \in B^{\mathrm{sm}},\right. \\
\xi_{j} & \left.=\xi_{j}\left(x_{1}, x_{2}\right) \text { for } j=1,2\right\},
\end{aligned}
$$


of the SYZ fibration $\rho: Y \rightarrow B$, its SYZ transform is the connection $\check{\nabla}$ which can locally be expressed as

$$
\check{\nabla}_{U}=d+2 \pi \sqrt{-1}\left(\xi_{1} d y_{1}+\xi_{2} d y_{2}\right)
$$

Let $\mathcal{L}$ be the line bundle determined by $\check{\nabla}$. Then the degree of its restriction to $E_{i}$ is given by

$$
\begin{aligned}
\left.\operatorname{deg} \mathcal{L}\right|_{E_{i}} & =\int_{E_{i}} \frac{\sqrt{-1}}{2 \pi} F_{\check{\nabla}} \\
& =-\int_{E_{i}}\left(d \xi_{1} \wedge d y_{1}\right) \\
& =-\left(\xi_{1}\left(s_{i}\right)-\xi_{1}\left(s_{i-1}\right)\right) .
\end{aligned}
$$

We have the second equality because $y_{2}$ is constant (and $x_{2}=-\lambda=0$ ) on $E_{i}$. Hence the isomorphism class of the line bundle $\mathcal{L}$ is completely determined by the increment of the angle coordinate $\xi_{1}$ on the Lagrangian section $L$ as one moves from $\left(s_{i-1}, 0\right)$ to $\left(s_{i}, 0\right)$.

Now we prove Theorem 1.1:

Proof of Theorem 1.1. Recall that the Hamiltonian isotopy class of the Lagrangian submanifold $L_{\gamma}$ remains unchanged when we deform $\gamma$ in a fixed homotopy class relative to the boundary conditions $\lim _{t \rightarrow-\infty}|\gamma(t)|=0$ and $\lim _{t \rightarrow \infty}|\gamma(t)|=\infty$. So, up to a re-parametrization of $\gamma$, we can deform the restriction of $\gamma$ to $\left(s_{i-1}, s_{i}\right)$ to a path arbitrary close to the concatenation of $\left.\gamma_{0}\right|_{\left(s_{i-1}, s_{i}\right)}$ (the negative axis) with a loop winding around the circle $l_{s}:=\{z \in$ $\left.\mathbb{C}^{\times}|| z \mid=\mathrm{e}^{s_{i}}\right\}$ for $w_{i}(\gamma)$ times. Along $\gamma_{0}$, the angle coordinate $\xi_{1}$ is constantly zero, and $\xi$ increases by one when we wind around $l_{s}$ once in the anticlockwise direction. Hence, the increment $\xi_{1}\left(s_{i}\right)-\xi_{1}\left(s_{i-1}\right)$ is precisely given by the $i$-th winding number $w_{i}(\gamma)$.

One way to visualize the Lagrangian submanifold $L_{\gamma}$ is to observe that the curve $\tau_{s_{0}}^{s_{i}}\left(C_{s_{0}}\right)$ is given by twisting $C_{s_{0}}$ in the vanishing cycle for $\sum_{k=1}^{i}$ $w_{i}(\gamma)$ times. Correspondingly, the increment of the angle coordinate $\xi_{1}$ as one goes from $\left(s_{i-1}, 0\right)$ to $\left(s_{i}, 0\right)$ is given by the $i$ th winding number $w_{i}(\gamma)$ of $\gamma$. 


\section{Homological mirror symmetry}

Let $\mathcal{X}$ be a smooth toric Fano stack of dimension $d$ and $\mathcal{K}$ be the total space of its canonical bundle. The mirror of $\mathcal{X}$ is given by a Laurent polynomial

$$
W:\left(\mathbb{C}^{\times}\right)^{d} \rightarrow \mathbb{C}
$$

whose Newton polytope coincides with the fan polytope of $\mathcal{X}$. By choosing sufficiently general $W$, one may assume that

- $W$ is tame in the sense that the gradient $\|\nabla W\|$ is bounded from below by a positive number outside of a compact set,

- all the critical points of $W$ are non-degenerate in the sense that the Hessian at each critical point is a non-degenerate quadratic form,

- all the critical values of $W$ are distinct, and

- the origin is not a critical value of $W$.

When $\mathcal{X}=\mathbb{P}(1, n)$ is the weighted projective line of weight $(1, n)$, one can take

$$
W: \mathbb{C}^{\times} \rightarrow \mathbb{C}, \quad z \mapsto z+1 / z^{n}
$$

as its mirror. This is related to the function $1 / z+z^{n}$ appearing in (1.6) by an automorphism $z \mapsto 1 / z$ of the torus, and our choice here is made only for aesthetic reason (figure 6 below looks nicer for this choice). The critical points of $W$ are given by $z=\sqrt[n+1]{n} \zeta_{n+1}^{i}, i=0, \ldots, n$, with critical values $\sqrt[n+1]{n}(1+1 / n) \zeta_{n+1}^{i}$. Here $\zeta_{n+1}=\exp (2 \pi \sqrt{-1} /(n+1))$ is a primitive $(n+1)$-st root of unity.

We equip $\left(\mathbb{C}^{\times}\right)^{d}$ with the Kähler form

$$
\omega=-\frac{\sqrt{-1}}{2}\left(\frac{d z_{1} \wedge d \overline{z_{1}}}{\left|z_{1}\right|^{2}}+\cdots+\frac{d z_{d} \wedge d \overline{z_{d}}}{\left|z_{d}\right|^{2}}\right),
$$

and define a horizontal distribution on $\left(\mathbb{C}^{\times}\right)^{d}$ as the orthogonal complement to the tangent spaces to fibers of $W$. Choose a sufficiently large closed disk $B \subset \mathbb{C}$ and a point $*$ on the boundary of $B$, so that all the critical values of $W$ is contained in the interior of $B$. The restriction $\left.W\right|_{S}: S \rightarrow B$ of $W$ to the intersection $S$ of $W^{-1}(B)$ and a sufficiently large closed ball in $\left(\mathbb{C}^{\times}\right)^{d}$ is a compact convex Lefschetz fibration [28, Definition 2.14], i.e., a family of compact convex symplectic manifolds with at worst non-degenerate critical points. Here, a compact convex symplectic manifold (also known as a 


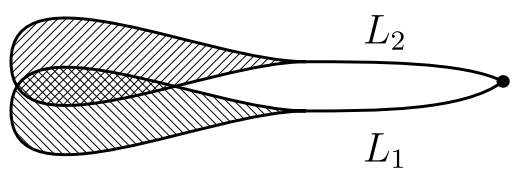

Figure 2: Admissible Lagrangians.

Liouville domain) is an exact symplectic manifold with boundary whose Liouville vector field points strictly outward along the boundary. We will write $\left.W\right|_{S}$ as $W$ by abuse of notation. By rounding the corners of $S$, one obtains a Liouville domain $M$. Its completion $\hat{M}=M \cup_{\partial M}(\partial M \times[1, \infty))$, obtained by gluing the positive half of the symplectization to the boundary, is symplectomorphic to $\left(\mathbb{C}^{\times}\right)^{d}$.

When $W=z+1 / z^{n}$, we fix a large positive real number $*$ as a base point, and let $B$ to be a closed disk of radius $*$ centered at the origin. Its inverse image $S:=W^{-1}(B)$ is symplectomorphic to a cylinder $[0, L] \times S^{1}$ for some $L$, equipped with the standard symplectic form $\omega=d r \wedge d \theta$. The fiber $S_{*}:=W^{-1}(*)$ consists of $n+1$ points, one of which is approximately $*$ and $n$ of which are approximately $n$-th roots of $1 / *$.

With a compact convex Lefschetz fibration, Abouzaid [2,3] associates a category $\mathcal{F}\left(S, S_{*}\right)$ consisting of exact Lagrangian submanifolds $L$ with boundaries, which are admissible in the sense that

- the boundary $\partial L$ is a Lagrangian submanifold in the interior of $S_{*}$,

- $L$ projects by $W$ to a curve $\gamma \subset B$ in a neighborhood of $\partial L$, and

- $L$ coincides with the parallel transport of $\partial L$ along $\gamma$ in this neighborhood.

See figure 2 for a schematic picture of admissible Lagrangians.

For a pair $\left(L_{1}, L_{2}\right)$ of admissible Lagrangian submanifolds, the space $\operatorname{hom}_{\mathcal{F}\left(S, S_{*}\right)}\left(L_{1}, L_{2}\right)$ of morphisms is defined as the Lagrangian intersection Floer complex $C F\left(L_{1}^{\epsilon_{1}}, L_{2}\right)$ between $L_{1}^{\epsilon_{1}}$ and $L_{2}$. Here $L_{1}^{\epsilon_{1}}$ is the Lagrangian submanifold obtained by perturbing the part of $L_{1}$ fibered over $\gamma_{1}$ to another Lagrangian submanifold fibered over the curve $\gamma_{1}^{\epsilon_{1}}$ starting from $*$ such that $\arg \gamma_{1}^{\epsilon_{1}}(0)<\arg \gamma_{2}^{\prime}(0)<\arg \gamma_{1}^{\epsilon_{1}}(0)+\pi$ as in figure 3 , and one only looks at intersection points in the interior of $S$. For a sequence $\left(L_{1}, \ldots, L_{k}\right)$ of admissible Lagrangians, the $A_{\infty}$-operation is defined by perturbing $L_{i}$ to $L_{i}^{\epsilon_{i}}$ such that $\arg \gamma_{1}^{\epsilon_{1}}(0)<\arg \gamma_{2}^{\epsilon_{2}}(0)<\cdots<\arg \gamma_{k}^{\epsilon_{k}}(0)<\arg \gamma_{1}^{\epsilon_{1}}(0)+\pi$ and counting virtual numbers of holomorphic disks bounded by these Lagrangian 


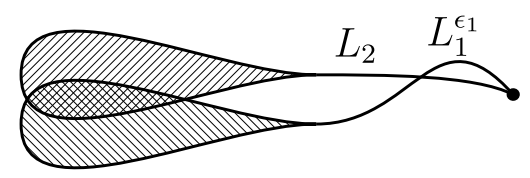

Figure 3: Perturbing $L_{1}$.

submanifolds. This gives an $A_{\infty}$-precategory, which can be promoted to an $A_{\infty}$-category by $[14$, Theorem 1.2$]$.

A vanishing path is an embedded path $\gamma:[0,1] \rightarrow B$ from the base point * to a critical value of $\pi$ avoiding other critical values. We assume that $\gamma$ does not pass through the origin. By arranging the vanishing cycles along a vanishing path, one obtains an admissible Lagrangian submanifold of $S$ called the Lefschetz thimble. A distinguished basis of vanishing paths is a sequence $\gamma=\left(\gamma_{1}, \ldots, \gamma_{m}\right)$ of mutually non-intersecting vanishing paths, one for each critical value and ordered according to $-\arg \gamma_{i}^{\prime}(0)$. The corresponding sequence of Lefschetz thimbles will be written as $\boldsymbol{\Delta}=\left(\Delta_{1}, \ldots, \Delta_{m}\right)$, and the full subcategory of $\mathcal{F}\left(S, S_{*}\right)$ consisting of $\boldsymbol{\Delta}$ will be denoted by $\mathcal{F}(\boldsymbol{\Delta})$. It is expected that

- $\boldsymbol{\Delta}$ is a full exceptional collection in $D^{b} \mathcal{F}\left(S, S_{*}\right)$, so that $D^{b} \mathcal{F}(\boldsymbol{\Delta})$ is equivalent to $D^{b} \mathcal{F}\left(S, S_{*}\right)$ and hence is independent of the choice of $\boldsymbol{\Delta}$, and

- $D^{b} \mathcal{F}\left(S, S_{*}\right)$ is equivalent to the derived category $D^{b} \mathcal{F}(W)$ of the Fukaya-Seidel category of $W$, defined in [32, Definition 18.12] as the $\mathbb{Z} / 2 \mathbb{Z}$-invariant part of the Fukaya category of a branched double cover of a slight enlargement of $S$.

Homological mirror symmetry for toric Fano stacks can be formulated as follows:

Conjecture 5.1 Kontsevich [23]. There exists an equivalence

$$
\Psi: D^{b} \mathcal{F}(\boldsymbol{\Delta}) \rightarrow D^{b} \operatorname{coh} \mathcal{X}
$$

of triangulated categories.

Let $\sigma: S \rightarrow S$ be a Hamiltonian diffeomorphism, which covers the Dehn twist $\bar{\sigma}: B \rightarrow B$ along a circle near the boundary of $B$. The resulting pushforward functor $\sigma_{*}: \mathcal{F}\left(S, S_{*}\right) \rightarrow \mathcal{F}\left(S, S_{*}\right)$ is an autoequivalence of the Fukaya category, which wraps a Lagrangian as shown in figure 4. 


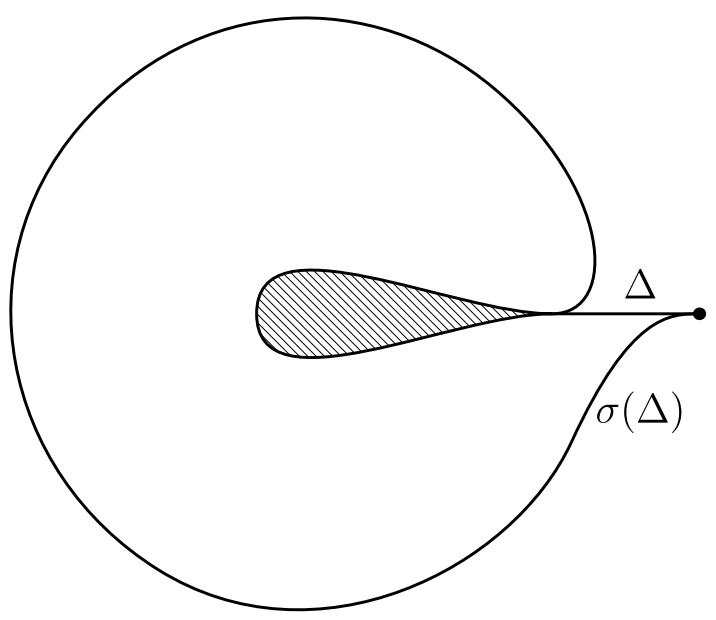

Figure 4: The functor $\sigma_{*}$.

One can equip the direct sum

$$
\mathcal{A}=\bigoplus_{i, j=0}^{m} \bigoplus_{k=0}^{\infty} \operatorname{Hom}_{\mathcal{F}\left(S, S_{*}\right)}\left(\sigma^{k}\left(\Delta_{i}\right), \Delta_{j}\right)
$$

with a ring structure given by

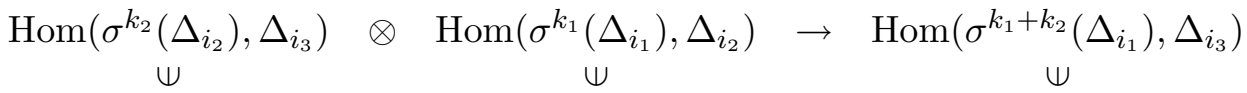

$$
\begin{aligned}
& x_{2} \\
& x_{1} \quad \mapsto \quad \mathfrak{m}_{2}\left(x_{2}, \sigma_{*}^{k_{2}}\left(x_{1}\right)\right) .
\end{aligned}
$$

The continuation map

$$
\mathfrak{t}: \operatorname{Hom}_{\mathcal{F}\left(S, S_{*}\right)}\left(\sigma^{k}\left(\Delta_{i}\right), \Delta_{j}\right) \rightarrow \operatorname{Hom}_{\mathcal{F}\left(S, S_{*}\right)}\left(\sigma^{k+1}\left(\Delta_{i}\right), \Delta_{j}\right)
$$

in Floer theory, obtained by counting solutions to inhomogeneous CauchyRiemann equation (cf. e.g [5, (3.35)]), induces an endomorphism $\mathfrak{T}$ of this ring.

On the mirror side, there is a distinguished autoequivalence

$$
S(-)=-\otimes \omega_{\mathcal{X}}[d]: D^{b} \operatorname{coh} \mathcal{X} \rightarrow D^{b} \operatorname{coh} \mathcal{X}
$$

called the Serre functor [8]. Let $s \in H^{0}\left(\omega_{\mathcal{X}}^{\vee}\right)$ be a section characterized by the property that the zero locus $s^{-1}(0)$ is the union of all the toric divisors 
of $\mathcal{X}$. This section induces a natural transformation

$$
\mathfrak{s}: S[-d] \rightarrow \text { id }
$$

which acts on objects by multiplication by $s: S[-d](X)=X \otimes \omega_{\mathcal{X}} \rightarrow$ id $(X)=X$. One can equip the direct sum

$$
\mathcal{B}=\bigoplus_{i, j=0}^{m} \bigoplus_{k=0}^{\infty} \operatorname{Hom}_{\mathcal{X}}\left(E_{i} \otimes \omega_{\mathcal{X}}^{k}, E_{j}\right)
$$

with a ring structure given by

$$
\begin{aligned}
\operatorname{Hom}\left(E_{i_{2}} \otimes \omega_{\mathcal{X}}^{k_{2}}, E_{i_{3}}\right) & \otimes \operatorname{Hom}\left(E_{i_{1}} \otimes \omega_{\mathcal{X}}^{k_{1}}, E_{i_{2}}\right) \\
& \stackrel{\sim}{\longrightarrow} \operatorname{Hom}\left(E_{i_{2}} \otimes \omega_{\mathcal{X}}^{k_{2}}, E_{i_{3}}\right) \\
& \otimes \operatorname{Hom}\left(E_{i_{1}} \otimes \omega_{\mathcal{X}}^{k_{1}+k_{2}}, E_{i_{2}} \otimes \omega_{\mathcal{X}}^{k_{2}}\right) \\
& \rightarrow \operatorname{Hom}\left(E_{i_{1}} \otimes \omega_{\mathcal{X}}^{k_{1}+k_{2}}, E_{i_{3}}\right) .
\end{aligned}
$$

The natural transformation (5.2) induces a ring endomorphism $\mathfrak{S}$ of $\mathcal{B}$.

Assume that the collection $\left(E_{i}\right)_{i=1}^{m}$ is cyclic in the sense that

$\operatorname{Ext}^{i}\left(E_{k} \otimes \omega_{\mathcal{X}}^{j}, E_{\ell}\right)=0$ for any $i \neq 0$, any $j \geq 0$ and any $k, \ell \in\{1, \ldots, m\}$.

This implies that the ring $\mathcal{B}$ is concentrated in degree zero, so that there are no higher $A_{\infty}$-operations for degree reason. Although cyclicity is a strong condition, it is known that any toric Fano stack in dimension two has a cyclic full exceptional collection of line bundles [21]. It is not known whether any toric Fano stack has a cyclic full exceptional collection of complexes.

Conjecture 5.2 Kontsevich, Seidel [33, Example 5]. There is a ring isomorphism $\mathcal{A} \stackrel{\sim}{\rightarrow} \mathcal{B}$ sending the ring endomorphism $\mathfrak{T}$ to $\mathfrak{S}$.

It is further expected that $t$ is the first component of a canonical natural transform

$$
\mathfrak{t}: \sigma_{*} \rightarrow \mathrm{id}
$$

which is mirror to the natural transformation (5.2).

When $W=z+1 / z^{n}$, we take a distinguished basis $\left(\gamma_{i}\right)_{i=0}^{n}$ of vanishing paths as in figure 5 . The corresponding Lefschetz thimbles $\left(\Delta_{i}\right)_{i=0}^{n}$ are Lagrangian submanifolds of $S$ with boundaries on $S_{*}$ as shown in Figure 6 . 


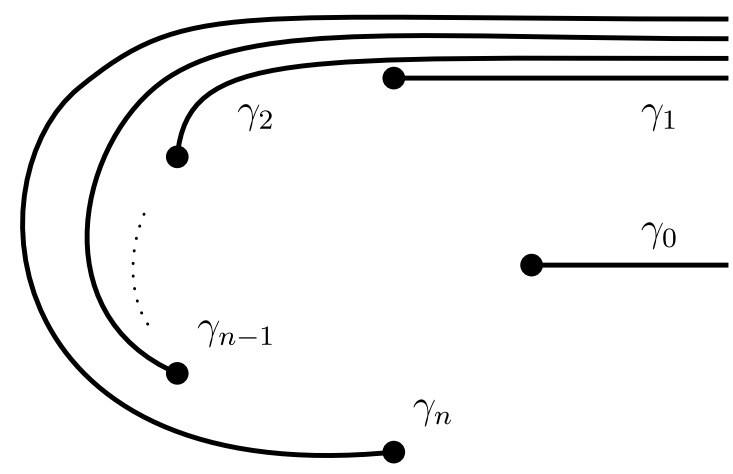

Figure 5: Vanishing paths on the $W$-plane.

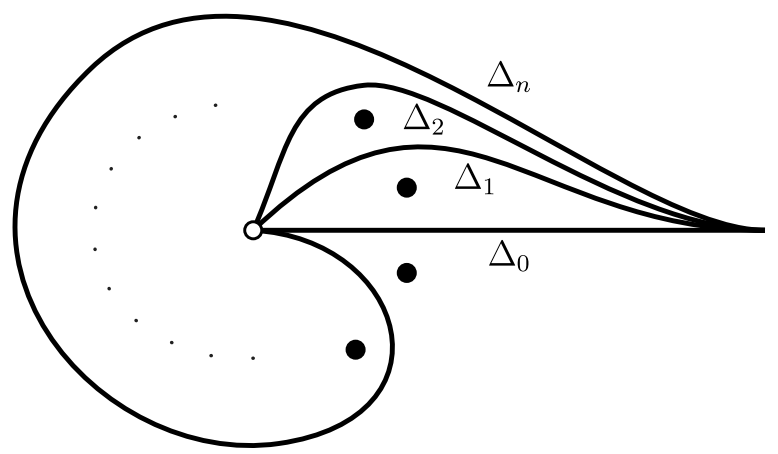

Figure 6: Lefschetz thimbles on the $z$-plane.

The Hamiltonian diffeomorphism $\sigma: S \rightarrow S$ behaves as $z \mapsto \exp (2 \pi \sqrt{-1}) z$ for $|z| \gg 1$, and $z \mapsto \exp (-2 \pi \sqrt{-1} / n) z$ for $|z| \ll 1$. We write the images of the Lefschetz thimbles as $\Delta_{-(n+1) k+i}=\sigma^{k}\left(\Delta_{i}\right)$.

On the mirror side, we write the homogeneous coordinate ring of $\mathcal{X}=$ $\mathbb{P}(1, n)$ as $\mathbb{C}[x, y]$ where $\operatorname{deg} x=1$ and $\operatorname{deg} y=n$. The canonical bundle is given by $\omega_{\mathcal{X}}=\mathcal{O}_{\mathcal{X}}(-n-1)$, and the full exceptional collection

$$
\left(E_{0}, E_{1}, \ldots, E_{n}\right)=\left(\mathcal{O}_{\mathcal{X}}, \mathcal{O}_{\mathcal{X}}(1), \ldots, \mathcal{O}_{\mathcal{X}}(n)\right)
$$

of line bundles is cyclic.

Theorem 5.3. Conjecture 5.2 holds for $\mathcal{X}=\mathbb{P}(1, n)$ and $W=z+1 / z^{n}$.

Proof. The Lefschetz thimble $\Delta_{-k(n+1)+i}$ starts from the critical point $\sqrt[n+1]{n} \zeta_{n+1}^{i}$ and extends in two directions: one wraps around the origin $k / n$ 


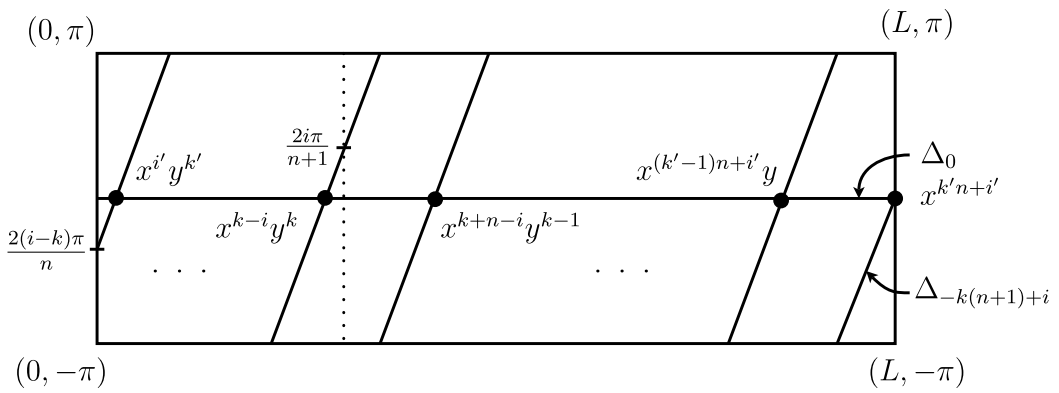

Figure 7: Intersections of Lefschetz thimbles.

times and goes to the point in $S_{*}$ approximated by $\zeta_{n}^{i-k} \cdot *^{-1 / n}$. The other wraps around infinity $k$ times and asymptotes to the point in $S_{*}$ approximated by $*$. Figure 7 shows the picture of two Lefschetz thimbles. Here the top and the bottom edges of the rectangle are identified to form the cylinder $[0, L] \times S^{1}$, which is symplectomorphic to $S$. The vertical dotted line shows the locus where the absolute value is $\sqrt[n+1]{n}$. The Lefschetz thimble $\Delta_{0}$ is just the real line, and the Lefschetz thimble $\Delta_{-k(n+1)+i}$ is obtained from the Lefschetz thimble $\Delta_{i}$ by wrapping $k$ times.

Write $k(n+1)-i=k^{\prime} n+i^{\prime}$ for $k^{\prime} \in \mathbb{Z}$ and $i^{\prime} \in\{0, \ldots, n-1\}$. Then the thimbles $\Delta_{-k(n+1)+i}$ and $\Delta_{0}$ intersect at $k^{\prime}+1$ points, and we label these intersection points by the basis of $\operatorname{Hom}\left(\mathcal{O}_{\mathbb{P}(1, n)}(-k(n+1)+i), \mathcal{O}_{\mathbb{P}(1, n)}\right)$ as shown in figure 7 . Intersections between other Lefschetz thimbles can be labeled similarly. By choosing the standard grading on $S$ determined by the holomorphic volume form $d z / z$ and suitable gradings on $\Delta_{i}$ 's, one can arrange that $\operatorname{Hom}_{\mathcal{F}\left(S, S_{*}\right)}\left(\Delta_{i}, \Delta_{j}\right)$ for $i \leq j$ have degree zero (and those for $i>j$ have degree one). It follows that the $\operatorname{ring} \mathcal{A}=\bigoplus_{i, j=0}^{m} \bigoplus_{k=0}^{\infty} \operatorname{Hom}_{\mathcal{F}\left(S, S_{*}\right)}$ $\left(\sigma^{k}\left(\Delta_{i}\right), \Delta_{j}\right)$ is concentrated in degree zero, so that there are no higher $A_{\infty^{-}}$ operations on $\mathcal{A}$. The multiplication on $\mathcal{A}$ comes from a triangle on $S$, which is either of the ones shown in figures 8 and 9 . This clearly matches the multiplication on $\mathcal{B}$ (which is just the multiplication of polynomials), and Theorem 5.3 is proved.

For $q \in \operatorname{Hom}_{\mathcal{F}\left(S, S_{*}\right)}\left(\sigma^{w}\left(\Delta_{i_{0}}\right), \Delta_{i_{1}}\right)$, we define ord $(q)$ as the maximal integer $d$ such that $x$ is in the image of

$$
\mathfrak{t}^{d}: \operatorname{Hom}_{\mathcal{F}\left(S, S_{*}\right)}\left(\sigma^{w-d}\left(\Delta_{i_{0}}\right), \Delta_{i_{1}}\right) \rightarrow \operatorname{Hom}_{\mathcal{F}\left(S, S_{*}\right)}\left(\sigma^{w}\left(\Delta_{i_{0}}\right), \Delta_{i_{1}}\right) .
$$

Proposition 5.4 below is an analogue of [29, Proposition 4.3], which will be useful later. 


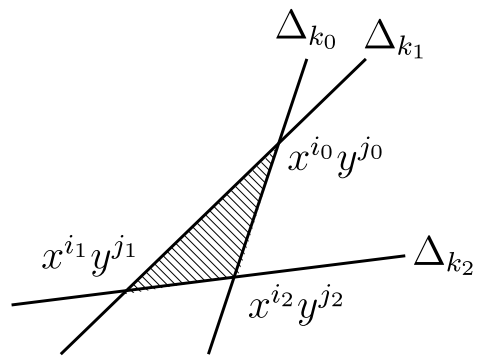

Figure 8: A triangle.

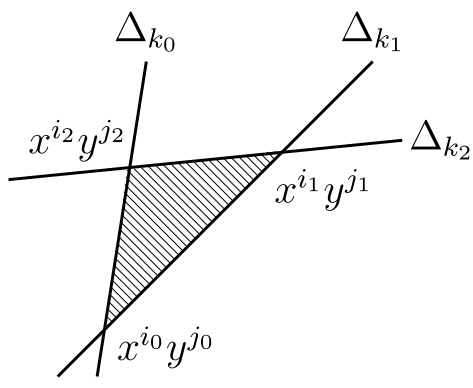

Figure 9: Another triangle.

Proposition 5.4. If a holomorphic triangle $\varphi: D^{2} \rightarrow S$ contributes to the composition $x^{i_{2}} y^{j_{2}}=\mathfrak{m}_{2}\left(x^{i_{1}} y^{j_{1}}, x^{i_{0}} y^{j_{0}}\right)$ for $x^{i_{0}} y^{j_{0}} \in \operatorname{Hom}_{\mathcal{F}\left(S, S_{*}\right)}\left(\Delta_{k_{0}}, \Delta_{k_{1}}\right)$, $x^{i_{1}} y^{j_{1}} \in \operatorname{Hom}_{\mathcal{F}\left(S, S_{*}\right)}\left(\Delta_{k_{1}}, \Delta_{k_{2}}\right)$, and $x^{i_{2}} y^{j_{2}} \in \operatorname{Hom}_{\mathcal{F}\left(S, S_{*}\right)}\left(\Delta_{k_{0}}, \Delta_{k_{2}}\right)$, then the intersection number between this triangle and the divisor $S_{0}$ is given by

$$
\varphi\left(D^{2}\right) \cdot S_{0}=\operatorname{ord}\left(x^{i_{2}} y^{j_{2}}\right)-\operatorname{ord}\left(x^{i_{0}} y^{i_{0}}\right)-\operatorname{ord}\left(x^{i_{1}} y^{j_{1}}\right)
$$

Proof. Since $\sqrt[n+1]{n}$ is close to 1 , one can perturb figure 7 slightly to set the dotted vertical line to be the unit circle on $\mathbb{C}^{\times}$without changing the intersections of triangles with $S_{0}$. Then $S_{0}$ is equidistributed on the dotted vertical line with vertical coordinates $(2 j+1) \pi /(2 n+2)$ for $j=0, \ldots, n$.

We write $d_{\ell}=\operatorname{ord}\left(x^{i_{\ell}} y^{j_{\ell}}\right)$ and $x^{i_{\ell}} y^{j_{\ell}}=(x y)^{d_{\ell}} x^{i_{\ell}-d_{\ell}} y^{j_{\ell}-d_{\ell}}$ for $\ell=0,1,2$. If $i_{\ell}-d_{\ell}=0$ for $\ell=0,1$ or $j_{\ell}-d_{\ell}=0$ for $\ell=0,1$, then the whole triangle is either on the left or on the right of the dotted vertical line in figure 7 , and both sides of (5.4) is zero. If $a:=i_{0}-d_{0}>0$ and $b:=j_{1}-d_{1}>0$, then the triangle $D$ looks as in figure 8 , and the vertical dotted line cuts $D$ into two. The number of points in $S_{0}$ on the part of the vertical dotted line bounded by $\Delta_{i_{0}}$ and $\Delta_{i_{1}}$ is given by $a$, and that by $\Delta_{i_{1}}$ and $\Delta_{i_{2}}$ is given by $b$ (here we are working on the universal cover of the cylinder $S$ ). The vertical dotted 
line is on the right or the left of the vertex $x^{i_{2}} y^{j_{2}}$ depending on whether $a>b$ or $a<b$ (and exactly on the line if $a=b$ ). It follows that both sides of (5.4) is given by $\max \{a, b\}$ in this case. The case $j_{0}-d_{0}>0$ and $i_{1}-d_{1}>0$ is similar, and Proposition 5.4 is proved.

An admissible Lagrangian submanifold $\Delta$ of $S$ can be completed to a Lagrangian submanifold $\hat{\Delta}:=\Delta \cup_{\partial \Delta}(\partial \Delta \times[1, \infty))$ of the completion $\hat{M}$. Given a pair $\left(\Delta_{1}, \Delta_{2}\right)$ of admissible Lagrangians, the wrapped Floer cohomology [5] is defined by

$$
H W\left(\hat{\Delta}_{1}, \hat{\Delta}_{2}\right):=\underset{w}{\lim } H F\left(\phi_{w}\left(\hat{\Delta}_{1}\right), \hat{\Delta}_{2}\right),
$$

where $\phi_{w}$ is the time $w$ flow by a Hamiltonian $H$ which behaves as $H(x, r)=$ $r$ on the cylindrical end $(x, r) \in \partial M \times[1, \infty)$ of $\hat{M}$.

Let

$$
Y^{\prime}:=\left\{(\boldsymbol{z}, u, v) \in\left(\mathbb{C}^{\times}\right)^{d} \times \mathbb{C}^{2} \mid W(\boldsymbol{z})=u v\right\}
$$

be the fiber of the double suspension of $W$.

Theorem 5.5 Seidel [34, Theorem 1]. Assume that the derived FukayaSeidel category $D^{b} \mathcal{F}(W)$ is quasi-equivalent to the derived category of coherent sheaves on $\mathcal{X}$ as an $A_{\infty}$-category. Then there exists a full embedding

$$
D^{b} \operatorname{coh}_{0} \mathcal{K} \hookrightarrow D^{b} \mathcal{F}\left(Y^{\prime}\right)
$$

of triangulated categories, where $D^{b} \operatorname{coh}_{0} \mathcal{K}$ is the full subcategory of $D^{b} \operatorname{coh} \mathcal{K}$ consisting of complexes whose cohomologies are supported on the zerosection, and $\mathcal{F}\left(Y^{\prime}\right)$ is the Fukaya category of $Y^{\prime}$.

The manifold $Y^{\prime}$ is an affine algebraic variety, and hence a Stein manifold of finite type, so that it is symplectomorphic to the completion $\hat{N}$ of the Liouville domain $N$ obtained by intersecting it with a sufficiently large ball. Consider the projection

$$
\begin{array}{cccc}
\varpi: & N & \rightarrow & \left(\mathbb{C}^{\times}\right)^{d} \\
\Psi & & \Psi \\
(\boldsymbol{z}, u, v) & \mapsto & \boldsymbol{z}
\end{array}
$$

and set $E=N \cap \varpi^{-1}(S)$. The restriction $\left.\varpi\right|_{E}: E \rightarrow S$ of the projection, which we will write $\varpi$ by abuse of notation, is a compact convex Lefschetz fibration, whose discriminant locus is $S_{0}:=W^{-1}(0)$. The completion $\hat{E}$ of 
$E$, obtained by first completing in the direction of the fiber of $\varpi$ and then extending it to the completion of the base $S$, is symplectomorphic to $Y^{\prime}$.

We define the Lagrangian submanifolds $L_{i} \subset E$ which are $\mathbb{R}$-fibrations over the Lefschetz thimbles $\Delta_{i} \subset S$ as in Section 3. When $W=z+1 / z^{n}$, the Lagrangian submanifold $L_{0}$ is given by

$$
L_{0}:=\left\{(z, u, v) \in E \mid z, u, v \in \mathbb{R}^{>0}\right\},
$$

and other Lagrangian submanifolds $L_{i}$ are given by $L_{i}=\zeta_{n+1}^{i} \cdot L_{0}$, where $\zeta_{n+1}^{i}$ acts on $E$ by sending $(z, u, v)$ to $\left(\zeta_{n+1}^{i} z, \zeta_{n+1}^{i} u, v\right)$.

Recall from [28, Definition 2.21] that a Lefschetz admissible Hamiltonian is a smooth function $H(\boldsymbol{z}, u, v)$ on $Y^{\prime}$, which can be written, outside of a compact set, as the sum $H_{b}(\boldsymbol{z})+H_{f}(u, v)$ of admissible Hamiltonians $H_{b}(\boldsymbol{z})$ and $H_{f}(u, v)$ on the base and the fiber respectively. The map $\varpi$ is not an honest Lefschetz fibration but a Bott-Morse analogue of a Lefschetz fibration in general. It is an honest Lefschetz fibration when $W=z+1 / z^{n}$ for dimensional reason.

The Lefschetz wrapped Floer cohomology is defined as the direct limit

$$
H W_{l}\left(\hat{L}_{i}, \hat{L}_{j}\right):=\underset{w}{\lim _{w}} H F\left(\phi_{w}\left(\hat{L}_{i}\right), \hat{L}_{j}\right)
$$

where $\phi_{w}: Y^{\prime} \rightarrow Y^{\prime}$ is the time $w$ flow defined by the Hamiltonian $H$. Set

$$
\mathscr{A}_{w}:=\bigoplus_{i, j=1}^{m} H F\left(\phi_{w}\left(\hat{L}_{i}\right), \hat{L}_{j}\right)
$$

and

$$
\mathscr{A}:=\lim _{w \rightarrow \infty} \mathscr{A}_{w}=\bigoplus_{i, j=1}^{m} H W_{l}\left(\hat{L}_{i}, \hat{L}_{j}\right) .
$$

The map

$$
\phi_{w+1, w}^{\mathscr{A}}: H F\left(\phi_{w}\left(\hat{L}_{i}\right), \hat{L}_{j}\right) \rightarrow H F\left(\phi_{w+1}\left(\hat{L}_{i}\right), \hat{L}_{j}\right)
$$

in the definition of the inductive limit is the continuation map for the Hamiltonian diffeomorphism $\phi_{1}: Y^{\prime} \rightarrow Y^{\prime}$. The universal map to the inductive 
limit is denoted by

$$
\phi_{\infty, w}^{\mathscr{A}}: H F\left(\phi_{w}\left(\hat{L}_{i}\right), \hat{L}_{j}\right) \rightarrow \mathscr{A}
$$

The $\mathbb{C}$-vector space $\mathscr{A}$ has a ring structure coming from

$$
\begin{array}{ccc}
H F\left(\phi_{w_{2}}\left(\hat{L}_{j}\right), \hat{L}_{k}\right) \otimes H F\left(\phi_{w_{1}}\left(\hat{L}_{i}\right), \hat{L}_{j}\right) & \rightarrow & H F\left(\phi_{w_{1}+w_{2}}\left(\hat{L}_{i}\right), \hat{L}_{j}\right) \\
\Psi & & \Psi \\
q \otimes r & \mapsto & \mathfrak{m}_{2}\left(q,\left(\phi_{w_{2}}\right)_{*}(r)\right) .
\end{array}
$$

One can show the ring isomorphism [12, Theorem A.2]

$$
\mathscr{A} \cong \bigoplus_{i_{0}, i_{1}=1}^{n} H W\left(\hat{L}_{i_{0}}, \hat{L}_{i_{1}}\right)
$$

with the ordinary wrapped Floer cohomology along the lines of $[28$, Theorem $2.22]$.

Note that the monodromy of the conic fibration $\varpi: E \rightarrow S$ around the discriminant $S_{0}$ is given by the Dehn twist along the vanishing cycle, which is inverse to the wrapping on the fiber. It follows that for a positive integer $w$, the intersection points $\phi_{w}\left(\hat{L}_{i_{0}}\right) \cap \hat{L}_{i_{1}}$ can be parametrized as $q_{j}$ for some $q \in \sigma^{w}\left(\Delta_{i_{0}}\right) \cap \Delta_{i_{1}}$ and an integer $j \in[0, \operatorname{ord}(q)+w]$ indicating the position in the fiber of $\varpi$ counted from the top as shown in figures 10 and 11 . The continuation map $\phi_{w+1, w}^{\mathscr{A}}: \mathscr{A}_{w} \rightarrow \mathscr{A}_{w+1}$ is written as $\phi_{w+1, w}^{\mathscr{A}}\left(q_{j}\right)=\mathfrak{t}(q)_{j+1}$ in this parametrization. We write $q=\mathfrak{t}^{\operatorname{ord}(q)}\left(q^{\prime}\right)$, and consider the $\mathbb{C}$-linear map

$$
\begin{aligned}
& \psi_{w}: \quad H F\left(\phi_{w}\left(\hat{L}_{i_{0}}\right), \hat{L}_{i_{1}}\right) \rightarrow \underset{\cup}{H} \rightarrow \underset{k=0}{\operatorname{Hom}} \underset{\mathcal{X}}{\left(E_{i_{0}} \otimes \omega_{\mathcal{X}}^{k}, E_{i_{1}}\right)} \\
& q_{j} \quad \mapsto \quad(s-1)^{j} Q^{\prime}
\end{aligned}
$$

where $Q^{\prime} \in \operatorname{Hom}_{\mathcal{X}}\left(E_{i_{0}} \otimes \omega_{\mathcal{X}}^{w-\operatorname{ord}(q)}, E_{i_{1}}\right)$ corresponds to $q^{\prime} \in \operatorname{Hom}_{\mathcal{F}\left(S, S_{*}\right)}$ $\left(\sigma^{w-\operatorname{ord}(q)}\left(\Delta_{i_{0}}\right), \Delta_{i_{1}}\right)$ under the ring isomorphism in Theorem 5.3.

Let

$$
\mathscr{B}:=\lim _{w \rightarrow \infty} \mathscr{B}_{w}, \quad \mathscr{B}_{w}:=\bigoplus_{k=0}^{2 w} \bigoplus_{i, j=0}^{n} \operatorname{Hom}_{\mathcal{X}}\left(E_{i} \otimes \omega_{\mathcal{X}}^{k}, E_{j}\right)
$$

be the direct limit of the right-hand side of (5.6) with respect to the map

$$
\phi_{w+1, w}^{\mathscr{B}}: \mathscr{B}_{w} \rightarrow \mathscr{B}_{w+1}
$$



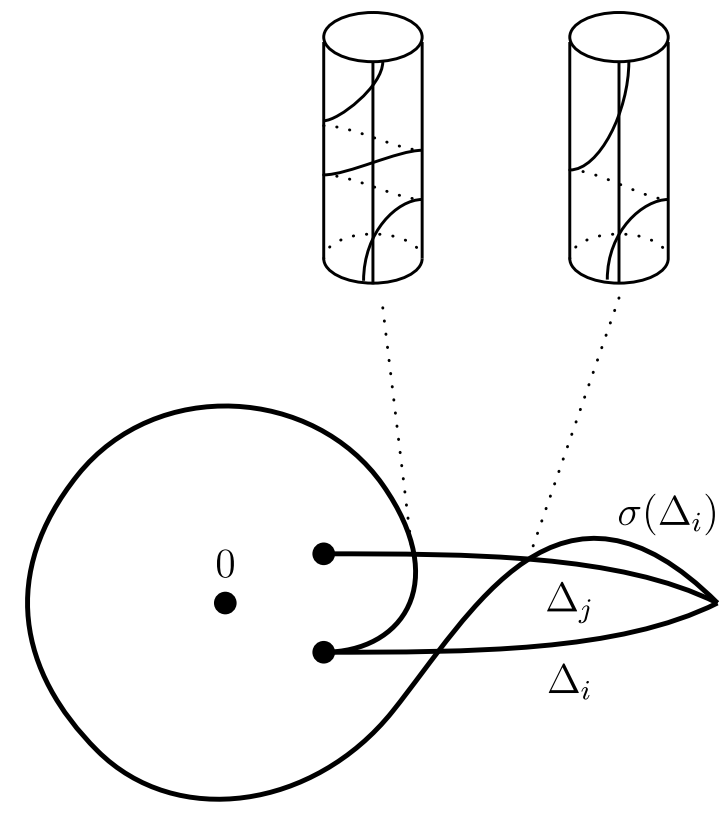

Figure 10: Wrapping once.

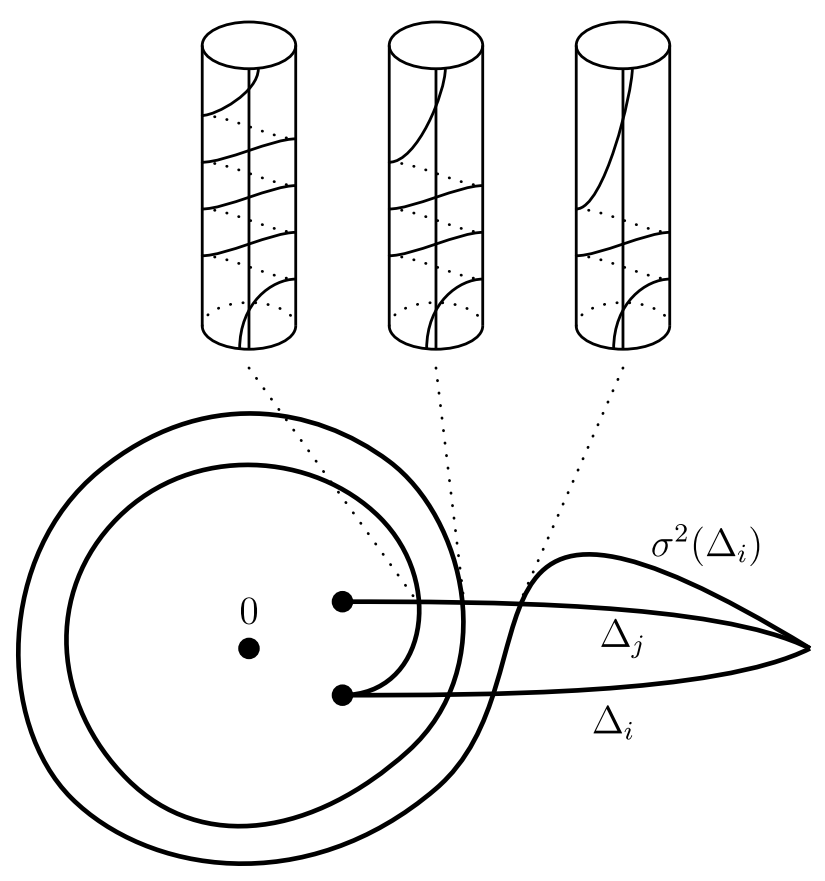

Figure 11: Wrapping twice. 
given by the multiplication by $1-s$

$$
\bigoplus_{k=0}^{2 w} \bigoplus_{i, j=0}^{n} \operatorname{Hom}_{\mathcal{X}}\left(E_{i} \otimes \omega_{\mathcal{X}}^{k}, E_{j}\right) \stackrel{1-s}{\longrightarrow} \bigoplus_{k=0}^{2 w+1} \bigoplus_{i, j=0}^{n} \operatorname{Hom}_{\mathcal{X}}\left(E_{i} \otimes \omega_{\mathcal{X}}^{k}, E_{j}\right)
$$

followed by the inclusion

$$
\bigoplus_{k=0}^{2 w+1} \bigoplus_{i, j=0}^{n} \operatorname{Hom}_{\mathcal{X}}\left(E_{i} \otimes \omega_{\mathcal{X}}^{k}, E_{j}\right) \hookrightarrow \bigoplus_{k=0}^{2 w+2} \bigoplus_{i, j=0}^{n} \operatorname{Hom}_{\mathcal{X}}\left(E_{i} \otimes \omega_{\mathcal{X}}^{k}, E_{j}\right)
$$

The multiplication in $\mathscr{B}$ is defined by

$$
\begin{array}{ccc}
\mathscr{B}_{w_{2}} \otimes \mathscr{B}_{w_{1}} & \rightarrow & \mathscr{B}_{w_{1}+w_{2}}, \\
\Psi & & \Psi \\
Q \otimes R & \mapsto & Q \cdot R .
\end{array}
$$

Proposition 5.6. One has an isomorphism

$$
\mathscr{B} \stackrel{\sim}{\longrightarrow} \bigoplus_{i, j=0}^{n} \operatorname{Hom}_{\check{\mathcal{Y}}}\left(\left.\pi^{*} E_{i}\right|_{\check{\mathcal{Y}}},\left.\pi^{*} E_{j}\right|_{\check{\mathcal{Y}}}\right)
$$

of rings.

Proof. By combining

$$
\begin{aligned}
\bigoplus_{i, j=0}^{n} \operatorname{Hom}_{\mathcal{K}}\left(\pi^{*} E_{i}, \pi^{*} E_{j}\right) & \cong \bigoplus_{i, j=0}^{n} \operatorname{Hom}_{\mathcal{X}}\left(E_{i}, \pi_{*} \pi^{*} E_{j}\right) \\
& \cong \bigoplus_{i, j=0}^{n} \operatorname{Hom}_{\mathcal{X}}\left(E_{i}, E_{j} \otimes \pi_{*} \mathcal{O}_{\mathcal{K}}\right) \\
& \cong \bigoplus_{i, j=0}^{n} \operatorname{Hom}_{\mathcal{X}}\left(E_{i}, E_{j} \otimes\left(\bigoplus_{k=0}^{\infty} \omega_{\mathcal{X}}^{-k}\right)\right) \\
& \cong \underset{w \rightarrow \infty}{\lim _{w \rightarrow 0}} \bigoplus_{k i, j=0}^{n} \bigoplus_{\mathcal{X}}^{n} \operatorname{Hom}_{\mathcal{X}}\left(E_{i} \otimes \omega_{\mathcal{X}}^{k}, E_{j}\right)
\end{aligned}
$$

with

$$
\operatorname{Hom}\left(\left.M\right|_{\check{\mathcal{Y}}},\left.N\right|_{\check{\mathcal{Y}}}\right)=\underline{\lim }(\operatorname{Hom}(M, N) \stackrel{1-s}{\longrightarrow} \operatorname{Hom}(M, N) \stackrel{1-s}{\longrightarrow} \cdots)
$$


for any objects $M$ and $N$ of $D^{b} \operatorname{coh} \mathcal{K}$ (cf. [31, (1.13)]), one obtains an isomorphism

$$
\bigoplus_{i, j=0}^{n} \operatorname{Hom}_{\check{\mathcal{Y}}}\left(\left.\pi^{*} E_{i}\right|_{\check{\mathcal{Y}}},\left.\pi^{*} E_{j}\right|_{\check{\mathcal{Y}}}\right) \cong \lim _{w \rightarrow \infty} \bigoplus_{k=0}^{2 w} \bigoplus_{i, j=0}^{n} \operatorname{Hom}_{\mathcal{X}}\left(E_{i} \otimes \omega_{\mathcal{X}}^{k}, E_{j}\right)
$$

of $\mathbb{C}$-vector spaces. The multiplication in $\mathscr{B}$ commutes with this $\mathbb{C}$-linear isomorphism, and Proposition 5.6 is proved.

Proposition 5.7. The maps $\psi_{w}$ are compatible with the composition, i.e.,

$$
\psi_{w_{1}+w_{2}}\left(\mathfrak{m}_{2}\left(q_{j}, r_{k}\right)\right)=\psi_{w_{2}}\left(q_{j}\right) \cdot \psi_{w_{1}}\left(r_{k}\right)
$$

for any $q_{j} \in \phi_{w_{2}}\left(\hat{L}_{i_{1}}\right) \cap \hat{L}_{i_{2}}$ and $r_{k} \in \phi_{w_{1}}\left(\hat{L}_{i_{0}}\right) \cap \hat{L}_{i_{1}}$.

Proof. Since the Lagrangian submanifolds $\hat{L}_{i}$ are fibered over $\Delta_{i}$, any holomorphic triangle in $\widetilde{\varphi}: D^{2} \rightarrow E$ contributing to $\mathfrak{m}_{2}\left(q_{j}, r_{k}\right)$ projects to a holomorphic triangle $\varphi=\varpi \circ \widetilde{\varphi}: D^{2} \rightarrow S$ contributing to $\mathfrak{m}_{2}(q, r)$. Given a holomorphic triangle $\varphi: D^{2} \rightarrow S$ contributing to $p=\mathfrak{m}_{2}(q, r)$, the contributions to $\mathfrak{m}_{2}\left(q_{j}, r_{k}\right)$ of holomorphic triangles $\widetilde{\varphi}: D^{2} \rightarrow E$ projecting to $\varphi$ is computed by Pascaleff [29, Proposition 4.4] as

$$
\mathfrak{m}_{2}\left(q_{j}, r_{k}\right)=\sum_{t=0}^{\ell}\left(\begin{array}{l}
\ell \\
t
\end{array}\right) p_{j+k+t}
$$

where $\ell=\varphi\left(D^{2}\right) \cdot S_{0}$ is the intersection number of the triangle and the discriminant $S_{0}$ of the fibration $\varpi: E \rightarrow S$. It follows that

$$
\begin{aligned}
\psi_{w_{1}+w_{2}}\left(\mathfrak{m}_{2}\left(q_{j}, r_{k}\right)\right) & =\sum_{t=0}^{\ell}\left(\begin{array}{l}
\ell \\
t
\end{array}\right) \psi_{w_{1}+w_{2}}\left(p_{j+k+t}\right) \\
& =\sum_{t=0}^{\ell}\left(\begin{array}{l}
\ell \\
t
\end{array}\right)(s-1)^{j+k+t} P^{\prime}
\end{aligned}
$$


On the right-hand side of (5.8), one has

$$
\begin{aligned}
\psi_{w_{2}}\left(q_{j}\right) \cdot \psi_{w_{1}}\left(r_{k}\right) & =(s-1)^{j} Q^{\prime} \cdot(s-1)^{k} R^{\prime} \\
& =(s-1)^{j+k} Q^{\prime} R^{\prime} \\
& =(s-1)^{j+k} s^{\ell^{\prime}} P^{\prime} \\
& =\sum_{t=0}^{\ell^{\prime}}\left(\begin{array}{c}
\ell^{\prime} \\
t
\end{array}\right)(s-1)^{j+k+t} P^{\prime}
\end{aligned}
$$

where $\ell^{\prime}=\operatorname{ord}(p)-\operatorname{ord}(q)-\operatorname{ord}(r)$. Now one has $\ell=\ell^{\prime}$ by Proposition 5.4, and Proposition 5.7 is proved.

Proposition 5.8. The maps $\psi_{w}$ induce on ismorphism $\psi: \mathscr{A} \stackrel{\sim}{\longrightarrow} \mathscr{B}$ of rings.

Proof. The maps $\psi_{w}$ induce a map $\psi: \mathscr{A} \rightarrow \mathscr{B}$ between inductive limits since

$$
\begin{aligned}
\psi_{w+1}\left(\phi_{w+1, w}^{\mathscr{A}}\left(q_{i}\right)\right) & =\psi_{w+1}\left(q_{i+1}\right) \\
& =(s-1)^{i+1} \cdot Q^{\prime} \\
& =\phi_{w+1, w}^{\mathscr{B}}\left((s-1)^{i} \cdot Q^{\prime}\right) \\
& =\phi_{w+1, w}^{\mathscr{B}}\left(\psi_{w}\left(q_{i}\right)\right) .
\end{aligned}
$$

For any element $q \in \operatorname{Hom}_{\check{\mathcal{Y}}}\left(\left.\pi^{*} E_{i}\right|_{\check{\mathcal{Y}}},\left.\pi^{*} E_{j}\right|_{\check{\mathcal{Y}}}\right)$, one can take sufficiently large $w_{1}$ so that $(s-1)^{w_{1}} q$ extends to an element of $\operatorname{Hom}_{\mathcal{K}}\left(\pi^{*} E_{i}, \pi^{*} E_{j}\right)$. Then one takes another sufficiently large $w_{2}$ so that $(s-1)^{w_{1}} q$ comes from $\bigoplus_{k=0}^{w_{2}} \operatorname{Hom}_{\mathcal{X}}\left(E_{i} \otimes \omega_{\mathcal{X}}^{k}, E_{j}\right) \subset \operatorname{Hom}_{\mathcal{K}}\left(\pi^{*} E_{i}, \pi^{*} E_{j}\right)$. Then $q$ is in the image of $\psi \circ \phi_{\infty, w_{1}+w_{2}}^{\mathscr{A}}: \mathscr{A}_{w_{1}+w_{2}} \rightarrow \mathscr{B}$, which shows that $\psi$ is surjective.

Note that the map $\phi_{\infty, w}^{\mathscr{B}}: \mathscr{B}_{w} \rightarrow \mathscr{B}$ is injective since each map $\phi_{w+1, w}^{\mathscr{B}}$ : $\mathscr{B}_{w} \rightarrow \mathscr{B}_{w+1}$ is injective. The map $\psi_{w}$ is also injective, and hence the map $\psi \circ \phi_{\infty, w}^{\mathscr{A}}=\phi_{\infty, w}^{\mathscr{B}} \circ \psi_{w}: \mathscr{A}_{w} \rightarrow \mathscr{B}$ is injective. This implies the injectivity of $\psi$, and Proposition 5.8 is proved.

Now we prove Theorem 1.2.

Proof of Theorem 1.2. Recall that an object $\mathcal{E}$ in a triangulated category is acyclic if $\operatorname{End}^{*}(\mathcal{E})$ is concentrated in degree zero. It is a generator if $\operatorname{Hom}(\mathcal{E}, X)=0$ implies $X \cong 0$. An acyclic generator is called a tilting object. Since the exceptional collection $\left(E_{i}\right)_{i=0}^{n}$ is full, the pull-back $\bigoplus_{i=0}^{n} \pi^{*} E_{i}$ is a generator of $D^{b} \operatorname{coh} \mathcal{K}$. Cyclicity of $\left(E_{i}\right)_{i=0}^{n}$ implies the acyclicity of $\bigoplus_{i=0}^{n} \pi^{*} E_{i}$. This shows that $\bigoplus_{i=0}^{n} \pi^{*} E_{i}$ is a tilting object. It follows that 


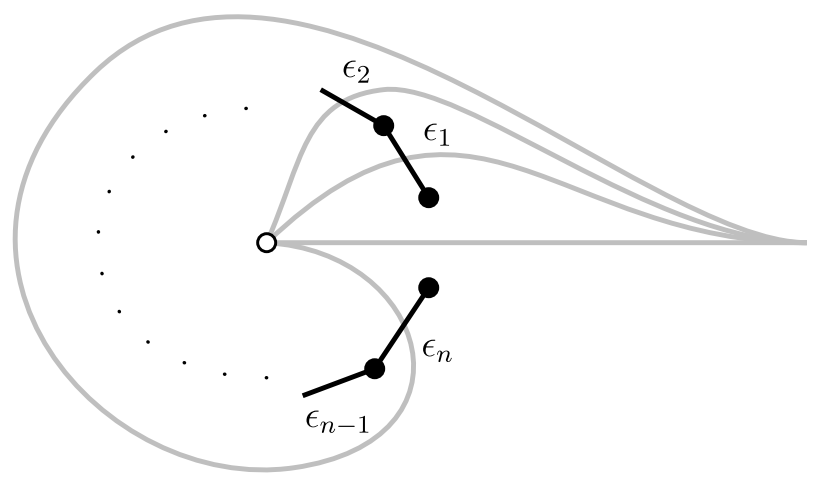

Figure 12: The dual cycles.

the restriction $\left.\bigoplus_{i=0}^{n} \pi^{*} E_{i}\right|_{\check{Y}}$ is a tilting object. (In general, the restriction of a generator to an open subset is a generator, and the restriction of an acyclic object to the complement of a principal divisor is acyclic.) Morita theory for derived categories $[9,30]$ shows that $D^{b} \operatorname{coh} \check{\mathcal{Y}}$ is equivalent to the derived category of finitely-generated modules over End $\left(\left.\bigoplus_{i=0}^{n} \pi^{*} E_{i}\right|_{\check{Y}}\right)$.

The direct sum $\bigoplus_{i} \hat{L}_{i}$ is acyclic by Proposition 5.8, and it is a generator of $\mathcal{W}^{\prime}$ by definition. It follows that $D^{b} \mathcal{W}^{\prime}$ is equivalent to to the derived category of finitely-generated modules over End $\left(\bigoplus_{i=0}^{n} \hat{L}_{i}\right)$, which is isomorphic to End $\left(\left.\bigoplus_{i=0}^{n} \pi^{*} E_{i}\right|_{\breve{Y}}\right)$ by Proposition 5.8. This concludes the proof of Theorem 1.2.

Finally, we discuss the compatibility of Theorem 1.1 and Theorem 1.2. Let $\left(\epsilon_{1}, \ldots, \epsilon_{n}\right)$ be the collection of line segments on $E$ connecting points in $W^{-1}(0)$ as in figure 12 , so that the intersection numbers with Lefschetz thimbles are given by

$$
\Delta_{i} \cdot \epsilon_{j}=\delta_{i j}, \quad i=0, \ldots, n, j=1, \ldots, n
$$

One can choose a symplectomorphism $Y \stackrel{\sim}{\longrightarrow} Y^{\prime}$ in such a way that these intersection numbers correspond precisely to the winding numbers in Definition 3.2. The SYZ transforms of the resulting Lagrangians in $Y$ are then given by line bundles $\left(\mathcal{L}_{i}\right)_{i=0}^{n}$ on $\check{Y}$ satisfying

$$
\left.\operatorname{deg} \mathcal{L}_{i}\right|_{E_{j}}=\delta_{i j}
$$

for $i=0, \ldots, n$ and $j=1, \ldots, n$. One can easily see, either from [26, Section 2] or by a direct calculation, that the endomorphism rings of $\left.\bigoplus_{i=0}^{n} \pi^{*} E_{i}\right|_{\check{Y}}$ and $\bigoplus_{i=0}^{n} \mathcal{L}_{i}$ are isomorphic, so that there is an equivalence 
$D^{b} \operatorname{coh} \check{\mathcal{Y}} \stackrel{\sim}{\longrightarrow} D^{b} \operatorname{coh} \check{Y}$ sending $\left.\pi^{*} E_{i}\right|_{\check{Y}}$ to $\mathcal{L}_{i}$. This gives the compatibility of Theorem 1.1 and Theorem 1.2 discussed in Introduction.

\section{Acknowledgments}

K. C. is supported by Hong Kong RGC Direct Grant for Research 2011/2012 (Project ID: CUHK2060434). K. U. is supported by JSPS Grant-in-Aid for Young Scientists No. 24740043. A part of this paper was worked out while K. U. was visiting the Chinese University of Hong Kong, whose hospitality is gratefully acknowledged.

\section{References}

[1] M. Abouzaid, D. Auroux and L. Katzarkov, Lagrangian fibrations on blowups of toric varieties and mirror symmetry for hypersurfaces, arXiv:1205.0053.

[2] M. Abouzaid, Homogeneous coordinate rings and mirror symmetry for toric varieties, Geom. Topol. 10 (2006), 1097-1157 (electronic).

[3] — Morse homology, tropical geometry, and homological mirror symmetry for toric varieties, Select. Math. (N.S.) 15(2) (2009), 189-270.

[4] D. Arinkin and A. Polishchuk, Fukaya category and Fourier transform, Winter School on Mirror Symmetry, Vector Bundles and Lagrangian Submanifolds (Cambridge, MA, 1999), AMS/IP Stud. Adv. Math., vol. 23, Amer. Math. Soc., Providence, RI, 2001, 261-274.

[5] M. Abouzaid and P. Seidel, An open string analogue of Viterbo functoriality, Geom. Topol. 14(2) (2010), 627-718.

[6] D. Auroux, Mirror symmetry and T-duality in the complement of an anticanonical divisor, J. Gökova Geom. Topol. GGT 1 (2007), 51-91.

[7] — Special Lagrangian fibrations, wall-crossing, and mirror symmetry, Surveys in differential geometry. Vol. XIII. Geometry, analysis, and algebraic geometry: forty years of the Journal of Differential Geometry, Surv. Differ. Geom., 13, Int. Press, Somerville, MA, 2009, 1-47.

[8] A.I. Bondal and M.M. Kapranov, Representable functors, Serre functors, and reconstructions, Izv. Akad. Nauk SSSR Ser. Mat. 53(6) (1989), 1183-1205, 1337. 
[9] A.I. Bondal, Representations of associative algebras and coherent sheaves, Izv. Akad. Nauk SSSR Ser. Mat. 53(1) (1989), 25-44.

[10] K. Chan, Homological mirror symmetry for $A_{n}$-resolutions as a T-duality, J. Lond. Math. Soc. (2) 87(1) (2013), 204-222.

[11] K. Chan, S.-C. Lau and N.C. Leung, SYZ mirror symmetry for toric Calabi-Yau manifolds, J. Different. Geom. 90(2) (2012), 177-250.

[12] K. Chan, D. Pomerleano and K. Ueda, Lagrangian torus fibrations and homological mirror symmetry for the conifold, arXiv:1305.0968.

[13] J.J. Duistermaat, On global action-angle coordinates, Comm. Pure Appl. Math. 33(6) (1980), 687-706.

[14] A.I. Efimov, A proof of the Kontsevich-Soibelman conjecture, Mat. Sb. 202(4) (2011), 65-84. MR 2830236 (2012m:53193)

[15] B. Fang, Homological mirror symmetry is T-duality for $\mathbb{P}^{n}$, Commun. Number Theory Phys. 2(4) (2008), 719-742.

[16] B. Fang, C.-C. Melissa Liu, D. Treumann and E. Zaslow, The coherentconstructible correspondence and homological mirror symmetry for toric varieties, Geometry and analysis. No. 2, Adv. Lect. Math. (ALM), vol. 18, Int. Press, Somerville, MA, 2011, 3-37.

[17] —, T-duality and homological mirror symmetry for toric varieties, Adv. Math. 229(3) (2012), 1875-1911.

[18] K, Fukaya, Multivalued Morse theory, asymptotic analysis and mirror symmetry, Graphs and patterns in mathematics and theoretical physics, Proc. Symp. Pure Math., 73, Amer. Math. Soc., Providence, RI, 2005, 205-278.

[19] M. Gross and B. Siebert, From real affine geometry to complex geometry, Ann. Math. (2) 174(3) (2011), 1301-1428.

[20] N. Hitchin, Lectures on special Lagrangian submanifolds, Winter School on Mirror Symmetry, Vector Bundles and Lagrangian Submanifolds (Cambridge, MA, 1999), AMS/IP Stud. Adv. Math., vol. 23, Amer. Math. Soc., Providence, RI, 2001, 151-182.

[21] A. Ishii and K. Ueda, Dimer models and exceptional collections, arXiv:0911.4529. 
[22] M. Kontsevich, Homological algebra of mirror symmetry, Proceedings of the International Congress of Mathematicians, 1, 2 (Zürich, 1994) (Basel), Birkhäuser, 1995, 120-139.

[23] —, Lectures at ENS Paris, spring 1998, set of notes taken by J. Bellaiche, J.-F. Dat, I. Martin, G. Rachinet and H. Randriambololona, 1998.

[24] M. Kontsevich and Y. Soibelman, Affine structures and nonArchimedean analytic spaces, The unity of mathematics, Progr. Math., 244, Birkhäuser Boston, Boston, MA, 2006, 321-385.

[25] - Notes on $A_{\infty}$ algebras, $A_{\infty}$ categories and non-commutative geometry. I, , 2006; arXiv:math/0606241.

[26] M. Kapranov and E. Vasserot, Kleinian singularities, derived categories and Hall algebras, Math. Ann. 316(3) (2000), 565-576.

[27] N.C. Leung, S.-T. Yau and E. Zaslow, From special Lagrangian to Hermitian-Yang-Mills via Fourier-Mukai transform, Adv. Theor. Math. Phys. 4(6) (2000), 1319-1341.

[28] M. McLean, Lefschetz fibrations and symplectic homology, Geom. Topol. 13(4) (2009), 1877-1944.

[29] J. Pascaleff, Floer cohomology in the mirror of the projective plane and a binodal cubic curve, arXiv:1109.3255.

[30] J. Rickard, Morita theory for derived categories, J. Lond. Math. Soc. (2) 39(3) (1989), 436-456.

[31] P. Seidel, $A_{\infty}$-subalgebras and natural transformations, Homology, Homotopy Appl. 10(2) (2008), 83-114.

[32] —- Fukaya categories and Picard-Lefschetz theory, Zurich Lectures in Advanced Mathematics, European Mathematical Society (EMS), Zürich, 2008.

[33] —, Symplectic homology as Hochschild homology, Algebraic geometry-Seattle 2005. Part 1, Proc. Sympos. Pure Math., vol. 80, Amer. Math. Soc., Providence, RI, 2009, 415-434.

[34] —, Suspending Lefschetz fibrations, with an application to local mirror symmetry, Comm. Math. Phys. 297(2) (2010), 515-528. 
[35] A. Strominger, S.-T. Yau and E. Zaslow, Mirror symmetry is T-duality, Nucl. Phys. B 479(1-2) (1996), 243-259.

Department of Mathematics

The Chinese University of Hong Kong

SHATIN

HONG KONG

E-mail address: kwchan@math.cuhk.edu.hk

Department of Mathematics

Graduate School of Science

OSAKA UNIVERSITY

MACHIKANEYAMA $1-1$

TOYONAKA

OSAKA 560-0043

JAPAN

E-mail address: kazushi@math.sci.osaka-u.ac.jp

ReCeIved November 12, 2012 